\title{
Xiaoyu Xiezhuo Drink Protects against Ischemia-Reperfusion Acute Kidney Injury in Aged Mice through Inhibiting the TGF- $\beta 1 /$ Smad3 and HIF1 Signaling Pathways
}

\author{
Qingqing Ye $\mathbb{D}$, Hongbo Chen, Hongzhen Ma, Xiaojun Xiang, Shouci Hu, Cong Xia, \\ and Lanjun Fu \\ Department of Nephrology, The First Affiliated Hospital of Zhejiang Chinese Medical University, Zhejiang, China \\ Correspondence should be addressed to Lanjun Fu; fulanjunzjtcm@126.com
}

Received 21 March 2021; Revised 2 August 2021; Accepted 14 August 2021; Published 10 September 2021

Academic Editor: Zhanjun Jia

Copyright (c) 2021 Qingqing Ye et al. This is an open access article distributed under the Creative Commons Attribution License, which permits unrestricted use, distribution, and reproduction in any medium, provided the original work is properly cited.

\begin{abstract}
Acute kidney injury (AKI) is responsible for significant mortality among hospitalized patients that is especially troubling aged people. An effective self-made Chinese medicine formula, Xiaoyu Xiezhuo Drink (XXD), displayed therapeutic effects on AKI. However, the compositions and underlying mechanisms of XXD remain to be elucidated. In this study, we used the ultra-highperformance liquid chromatography method coupled with hybrid triple quadrupole time-of-flight mass spectrometry (UHPLCQ-TOF-MS) to investigate the chemical components in XXD. Then, the absorbable components of XXD were identified based on the five principles and inputted into the SwissTargetPrediction and STITCH databases to identify the drug targets. AKIrelated targets were collected from the GenCLiP 3, GeneCards, and DisGeNET databases. The crossover genes of XXD and AKI were identified for functional enrichment analysis. The protein-protein interaction (PPI) network of crossover genes was constructed, followed by the identification of hub genes. Subsequently, the effects and potential mechanisms of XXD on AKI predicted by the network pharmacology and bioinformatics analyses were experimentally validated in ischemia-reperfusion (I/R) injury-induced AKI aged mouse models. A total of 122 components in XXD were obtained; among them, 58 components were found that could be absorbed in the blood. There were 800 potential drug targets predicted from the 58 absorbable components in AKI which shared 36 crossover genes with AKI-related targets. The results of functional enrichment analysis indicated that crossover genes mostly associated with the response to oxidative stress and the HIF1 signaling pathway. In the PPI network analysis, 12 hub genes were identified, including ALB, IL-6, TNF, TP53, VEGFA, PTGS2, TLR4, NOS3, EGFR, PPARG, HIF1A, and HMOX1. In AKI aged mice, XXD prominently alleviated I/R injury-induced renal dysfunction, abnormal renal pathological changes, and cellular senescence, inflammation, and oxidative damage with a reduction in the expression level of the inflammatory mediator, $\alpha$-SMA, collagen-1, F4/80, TP53, VEGFA, PTGS2, TLR4, NOS3, EGFR, PPARG, HIF1A, ICAM-1, TGF- $\beta 1$, Smad3, and p-Smad3 and an increase of nephridial tissue p-H3, Ki67, HMOX1, MMP-9, and Smad7 levels. In summary, our findings suggest that XXD has renoprotective effects against AKI in aged mice via inhibiting the TGF$\beta 1 /$ Smad 3 and HIF1 signaling pathways.
\end{abstract}

\section{Introduction}

Acute kidney injury (AKI), previously called acute renal failure (ARF), is a serious clinical disease characterized by a rapid increase in serum creatinine and a rapid decline in glomerular filtration rate (GFR) $[1,2]$. Because of an aging population, it can be seen in up to $7 \%$ of hospital admissions and $30 \%$ of
ICU admissions [3]. Moreover, it has been demonstrated that AKI leads to the accumulation of water, sodium, several electrolyte disturbances, renal fibrosis, and, eventually, chronic kidney disease (CKD) [3, 4]. Significantly, the long-term prognosis of AKI, especially in the elderly, is not optimistic. Compared with younger people, a higher incidence of AKI was observed in older adults $[5,6]$. Additionally, the epidemiological study 
of AKI in China showed that persons over 60 years old were accounted for $57.73 \%$ among 7604 AKI patients [7]. Thus, novel effective treatment strategies for AKI in older patients are still desired.

Ischemia is considered as the main cause that contributes to the development of AKI [8]. Ischemia-reperfusion (I/R) injury is tissue damage that can result from blood reperfusion, and I/R injury of kidney tissue is a common reason for AKI progression $[2,8]$. Besides, the pathophysiology of AKI to CKD transition is complex, and many factors such as cell senescence, apoptosis, inflammatory processes, and oxidative stress are involved [9]. Aging is a process in which the cell structure and function of various tissues and organs in the body undergo decay and change with age under the influence of long-term internal and external environments [10]. Also, aging leads to the reduction of the kidney's resistance to (I/R) injury, drugs, contrast agents, and other poisons, so the elderly are more likely to develop AKI [11]. Renal fibrosis is the main manifestation of AKI and renal cell senescence. The process of cell senescence is accompanied by a series of changes in gene and protein expression in the aging kidney. I/R injury also exaggerates metabolic imbalance that leads to inflammation with the upregulation of proinflammatory mediators including interleukin 6 (IL-6), tumor necrosis factor $\alpha$ (TNF- $\alpha$ ), intercellular adhesion molecule (ICAM-1), transforming growth factor $\beta$ (TGF- $\beta$ ), and metalloproteinase 7/9 (MMP-7/9) [12, 13]. Furthermore, the p53/p21 pathway was activated in the renal tubular cells under the condition of acute stress, which blocks the cell cycle at the G1/S phase $[14,15]$. Therefore, these studies suggested that $\mathrm{I} / \mathrm{R}$ injury and renal fibrosis might serve as potential therapeutic targets for AKI in elderly men.

Traditional Chinese medicine (TCM) has attracted wide attention for the clinical treatment of AKI [16]. For instance, the ethanol extract of root bark of Illicium henryi was significantly dose-dependently against AKI in mice by inhibiting inflammatory responses and oxidative stress [17]. Xiaoyu Xiezhuo Drink (XXD) consists of Astragalus membranaceus (15g), processed Radix cyathulae (6g), Peach Kernel (6g), Lumbricus (6g), prepared Rhubarb (5g), and Plantain $(10 \mathrm{~g})$. It is derived from the "Buyang Huanwu Decoction" in the book of "Yilin Gaicuo" by Wang Qingren in the Qing Dynasty. Previous studies have shown that XXD is effective in treating patients with AKI. Animal studies have shown that XXD can alleviate pathological damage, reduce the protein expression levels of $\alpha$-smooth muscle actin ( $\alpha$-SMA), TGF- $\beta 1$, and collagen-1, as well as upregulate Smad7 levels in the kidney tissues of mice with unilateral ureteral occlusion (UUO) [18, 19]. Taken together, these data indicated that XXD treatment markedly suppressed renal interstitial fibrosis by regulating the TGF- $\beta /$ Smad singling pathway. However, the mechanism of XXD in the improvement of the aging kidney with I/R injury is unclear.

In recent years, the network pharmacology, first proposed by Hopkins in 2007 [20], was used as a promising strategy to investigate TCM from a system perspective based on system biology, bioinformatics, and high-throughput histology [21]. $\mathrm{XXD}$ is composed of many herbs, which result in a complex chemical composition. Therefore, it is difficult to fully understand their therapeutic mechanisms. Thus, it is necessary to extend the understanding of the antifibrosis effects of XXD in treating AKI of the elderly with I/R injury by using scientific and technologic approaches. In the present study, the components of the XXD were identified with ultra-high-performance liquid chromatography coupled with UHPLC-Q-TOF-MS analysis. Then, network pharmacology was used herein to predict the targets of XXD and AKI. Finally, corresponding experiments were then performed to confirm the protective effects of XXD and verify the results of prediction in aged mice with AKI. Our study is the first to identify potential absorbable compounds in XXD and elucidate their mechanisms in AKI treatment by using the network pharmacology approach.

\section{Material and Methods}

2.1. Preparation of XXD Aqueous Extracts. The XXD aqueous extracts were provided by the dispensary TCM of the First Affiliated Hospital of Zhejiang Chinese Medical University (Zhejiang, China). Briefly, $48 \mathrm{~g}$ of dried herbs of XXD was boiled with $1000 \mathrm{~mL}$ of distilled water. The mixture was gradually heated to $100^{\circ} \mathrm{C}$ over a period of $60 \mathrm{~min}$. And, the extracts were concentrated to $125 \mathrm{~mL}$ under reduced pressure in a rotavapor at $45^{\circ} \mathrm{C}$ to obtain an extract with a final concentration of $384 \mathrm{mg} / \mathrm{mL}$, according to the weight of initially dried herbs.

2.2. UHPLC-Q-TOF-MS Analysis. A Waters ACQUITY IClass Plus UPLC system (Waters Corporation, Milford, MA, USA) together with a SCIEX X-500R mass spectrometer (AB SCIEX, USA) was used for UHPLC-Q-TOF-MS analysis. Briefly, the above-mentioned $1.0 \mathrm{~mL}$ sample was well mixed in $1.0 \mathrm{~mL}$ analytical methanol. The mixed solutions were centrifuged at 14,000 rpm for $20 \mathrm{~min}$, and then, the supernatant was collected before analysis. Chromatographic separations were performed on an ACQUITY UPLC BEH C18 $(100 \mathrm{~mm} \times 2.1 \mathrm{~mm}, 1.7 \mu \mathrm{m})$. The mobile phase consisted of acetonitrile containing $0.1 \%$ formic acid (A) and $0.1 \%$ formic acid (B). Also, the gradient elution procedures were as follows: 0 21 $\mathrm{min}, 99 \% \sim 50 \% \mathrm{~A} ; 21 \sim 25.5$ $\min , 50 \% \sim 15 \% \mathrm{~A}$; and $25.5 \sim 27 \mathrm{~min}, 15 \% \sim 1 \% \mathrm{~A}$. The flow rate was set to $0.3 \mathrm{~mL} / \mathrm{min}$, and the injection volume was 2 $\mu \mathrm{L}$. The autosampler was maintained at $8^{\circ} \mathrm{C}$. A TurbolonSpray electrospray ionization (ESI) was used in positive and negative ion scanning modes. The operating parameters were optimized as follows: ion source gas 1 (Gas1): 45, ion source gas 2 (Gas2): 55, curtain gas (CUR): 35 , source temperature: $600^{\circ} \mathrm{C}$, IonSapary Voltage Floating (ISVF): 5500 $\mathrm{V}\left(\mathrm{ESI}^{+}\right)$or $-4500 \mathrm{~V}\left(\mathrm{ESI}^{-}\right)$; TOF $\mathrm{MS}$ scan at a range of 100-1500 Da, production scan at a range of 25-1500 Da, TOF MS scan accumulation time: 0.25 s/spectra, and product ion scan accumulation time: 0.035 s/spectra. Secondary mass spectrometry was obtained by using informationdependent acquisition (IDA) with a high sensitivity mode. The parameters of IDA were optimized as follows: collision energy: $35 \pm 15 \mathrm{eV}$, exclude isotopes within $4 \mathrm{Da}$, and candidate ions to monitor per cycle: 12 . The accurate mass and elemental composition for the precursor ions and fragment ions were analyzed by the SCIEX OS software which has a self-contained TCM MS/MS library. 
2.3. Identification of Absorbable Components of XXD. First, the molecular formulas of the chemical components were retrieved from the PubChem (https://pubchem.ncbi.nlm .nih.gov/) database that quickly finds chemical information from authoritative sources [22], and the canonical SMILES (simplified molecular-input line-entry system) format of a specific structural formula was acquired. Then, the Molinspiration SMILES online platform (https://www.molinspiration.com/) was employed to calculate the molecular properties and bioactivity score by using the SMILES format of the chemical components. When a chemical component met the basic requirements of the related parameters, based on the five principles of drug absorption, it was considered to be the absorbable component: relative molecular mass $\mathrm{MW} \leq 500$, hydrogen bond acceptor (the number of $\mathrm{O}$ and $\mathrm{N}$ ) $\mathrm{nON} \leq 10$, hydrogen bond donor (the number of hydrogen atoms attached to the $\mathrm{O}$ and $\mathrm{N}) \mathrm{nOHNH} \leq 5$, and fat water partition coefficient $\operatorname{miLogP} \leq 5$.

2.4. Prediction of Drug Targets for XXD. The potential protein targets of the absorbable components in XXD were retrieved from a web tool, SwissTargetPrediction (http:// www.swisstargetprediction.ch/), wherein the predictions are based on the similarity principle, through reverse screening [23], and the STITCH database (version 5.0, http://stitch .embl.de/) with the species selected as "H. sapiens" [24]. Only the protein targets which had direct interactions with each absorbable component in XXD were saved as the potential targets. After integration of potential protein targets, the absorbable component-target network was generated using Cytoscape software (version 3.6.1, https://cytoscape.org/, Boston, MA, USA) [25].

2.5. Collection of AKI-Related Targets. Related targets of AKI were collected from the GenCLiP 3 (http://ci.smu.edu.cn/ genclip3/analysis.php) [26], GeneCards (version 5.0, https:// www.genecards.org/) [27], and DisGeNET (version 7.0, http:// www.disgenet.org/) [28] databases with the keyword "Acute kidney injury."

2.6. Functional Enrichment and Protein-Protein Interaction (PPI) Network Analysis. Based on the previous steps, 4 sets of target lists were prepared. Then, we matched the potential drug targets of XXD absorbable components and the AKIrelated targets using the Venny 2.1 (https://bioinfogp.cnb .csic.es/tools/venny/index.html); then, the crossover genes were chosen as the potential targets of XXD in treating AKI. The Metascape (http://metascape.org/gp/index.html\#/ main/step1), a gene annotation and analysis interactive platform [29], was used for Gene Ontology- (GO-) biological process (BP) enrichment and Kyoto Encyclopedia of Genes and Genomes (KEGG) pathway enrichment analyses on the crossover genes. To further investigate the pharmacological mechanisms of XXD on AKI, the crossover genes were then processed by the STRING database to construct a PPI network (version 11.0, https://string-db.org/) [30], with species limited to "H. sapiens" and a confidence score $\geq 0.7$ and visualized by Cytoscape. Additionally, topological features of the PPI network were analyzed using the plug-in NetworkA- nalyzer. The node size was visualized by the degree value, meaning the number of combined crossover genes, and the degree greater than the median degree of all nodes was selected as the core target for treating AKI.

2.7. Experimental Animals and Treatment. Fifty healthy eighteen- to twenty-month-old male C57BL/6 mice (approximately 27 32 g) were purchased from Vital River Laboratory Animal Co., Ltd. (License number: SCXK (Beijing) 20120001; Beijing, China) and housed under standard conditions with a $12 \mathrm{~h}$ light-dark cycle, humidity of $50 \% \pm 10 \%$, and temperature of $24 \pm 1^{\circ} \mathrm{C}$ at the Animal Center of Zhejiang Chinese Medicine University. All experimental procedures were approved by the Zhejiang Chinese Medical University and were conducted according to the Guide for the Care and Use of Laboratory Animal of the National Institute of Health (Publication No. 80-23, revised 1996). All mice had free access to food and water during the experiments. Also, mice were acclimatized for 1 week before use. According to previously published methods by Arfian et al. [13], bilateral renal pedicle clamping was conducted to induce kidney $I / R$ injury in this study. Briefly, the mice were intraperitoneally injected with sodium pentobarbital $(0.1 \mathrm{~mL} / 10 \mathrm{~g}$ body weight). After being anesthetized, the abdomen was opened. Then, both renal pedicles were exposed and clamped with a nontraumatic vascular clamp for 30 minutes, which was then removed. After the operating day, the mice were weighed and divided into five groups $(n=10)$ : the control group, the model group, and the XXD groups $(7.28 \mathrm{~g} / \mathrm{kg}, 14.56 \mathrm{~g} / \mathrm{kg}$, and $29.12 \mathrm{~g} / \mathrm{kg})$. Besides, the sham operation procedure was used for the control group with only opening of the abdomen of the mice without renal pedicle clamping. The mice in the XXD groups were administrated with XXD at the doses of $7.28 \mathrm{~g}$ raw herbs $/ \mathrm{kg}, 14.56 \mathrm{~g}$ raw herbs $/ \mathrm{kg}$, and $29.12 \mathrm{~g}$ raw herbs $/ \mathrm{kg}(0.1 \mathrm{~mL} / 10 \mathrm{~g}$ of mouse weight) by oral gavage every day for 14 days. The dosage for mice was $7.28 \mathrm{~g}$ raw herbs/ $\mathrm{kg}$ according to the human mouse equivalent dosage conversion of human clinical dosage $(48 \mathrm{~g} / 60 \mathrm{~kg} /$ day). The mice in the control and model groups were given an equal volume of $0.9 \%$ physiological saline. At the end of the experiment, the urine was collected. After the final administration for 2 hours, all mice were weighed and anesthetized by sodium pentobarbital. Then, the blood samples were collected from the eyes, and the serum was obtained by centrifuging at $3000 \mathrm{rpm}$ for $15 \mathrm{~min}$ at $4^{\circ} \mathrm{C}$. Subsequently, all the mice were sacrificed via cervical dislocation, and the renal tissues were removed, weighed, and stored at $-80^{\circ} \mathrm{C}$ for subsequent analyses. Furthermore, the renal index was calculated by using the following formula: kidney weight/mouse body weight $\times 100(\mathrm{~g} / 100 \mathrm{~g}$ body weight $)$.

2.8. Liver and Renal Function Assessment. Liver function was assessed by serum aspartate aminotransferase (AST) and alanine aminotransferase (ALT). Meanwhile, renal function was evaluated by serum creatinine (Cre), blood urea nitrogen (BUN), and urinary albumin (ALB) on a Hitachi 7180 automatic biochemical analyzer (Kyoto, Japan).

2.9. Histology Assessment and SA- $\beta$-gal Staining. To evaluate histological changes, a part of kidney tissues was fixed in $4 \%$ 
paraformaldehyde. After that, the samples were embedded in paraffin and cut into $4 \mu \mathrm{m}$ thick sections for hematoxylin and eosin (HE) and Masson staining for histological analysis and renal interstitial fibrosis observation, respectively. Also, SA- $\beta$-gal staining analysis was performed to assess the cellular senescence in kidney tissues of I/R-induced AKI aged mice with the SA- $\beta$-gal staining kit (ab65351, Abcam, Cambridge, MA), according to the manufacturer's protocol. Finally, the images were captured by optical microscopy (DM3000, Leica, Germany) with 200x and 400x magnifications.

2.10. Enzyme-Linked Immunosorbent Assay (ELISA). Oxidative stress levels of kidney tissues were assessed using malondialdehyde (MDA), superoxide dismutase (SOD), and glutathione (GSH) activities. Briefly, renal tissue samples (100 mg) were mixed with $900 \mu \mathrm{L}$ of PBS to prepare $10 \%$ homogenate by a tissue homogenizer (Shanghai, China). Then, the homogenate supernatants were separated by centrifuging at $12,000 \mathrm{rpm}$ for $20 \mathrm{~min}$ at $4^{\circ} \mathrm{C}$. Meanwhile, the protein concentration of the supernatants was quantified using the BCA Protein Assay Kit (Beyotime, China). The collected supernatants were measured for renal MDA, SOD, and GSH levels using commercial kits (Nanjing Jiancheng Bioengineering Institute, Nanjing, Jiangsu, China) based on the manufacturer's instruction.

2.11. Immunohistochemistry. The expression of $\alpha$-SMA, collagen-1, classic renal macrophage marker (F4/80), phosphohistone $\mathrm{H} 3$ (p-H3), and proliferation marker (Ki67) was assessed by immunohistochemical staining. Briefly, 4 sections were deparaffinized, and endogenous peroxidase activity was blocked by incubating in $3 \%$ hydrogen peroxide at $37^{\circ} \mathrm{C}$ for $30 \mathrm{~min}$. Then, the sections were incubated with $5 \%$ BSA for $30 \mathrm{~min}$ at $37^{\circ} \mathrm{C}$ to block the nonspecific antibody binding. Primary antibody of $\alpha$-SMA (1:500, AF1032, Affinity Biosciences, USA), collagen-1 ( 1 : 500, ab270993, Abcam, USA), F4/80 (200 $\mu \mathrm{g} / \mathrm{mL}$, sc-377009, Santa Cruz Biotechnology (Shanghai) Co., Ltd., USA), p-H3 (1:300, H6409, MilliporeSigma, Germany), and Ki67 (5 $\mu \mathrm{g} / \mathrm{mL}$, ab15580, Abcam, USA) was applied to the sections and incubated overnight at $4^{\circ} \mathrm{C}$. After washing three times with PBS, the sections were incubated with biotinylated goat anti-mouse/rabbit IgG $(0.5 \mu \mathrm{g} / \mathrm{mL}$; Abcam $)$ for $1 \mathrm{~h}$ at room temperature and stained using 3,3'-diaminobenzidine dihydrochloride. Digital images of immunohistochemistry staining were captured with the light microscope and $\alpha$-SMA, collagen-1, F4/80, p-H3, and Ki67 expressions were quantified with ImageJ software (National Institutes of Health, NY).

2.12. Quantitative Real-Time Polymerase Chain Reaction (PCR) Analysis. Total RNAs were isolated from renal tissues using a TRIzol Reagent (Invitrogen) and quantified by a NanoDrop 2000/2000c spectrophotometer (Thermo Fisher Scientific, Waltham, MA, USA). The complementary DNA (cDNA) was synthesized with the $1 \mu \mathrm{g}$ total RNA and a reverse transcription kit (TaKaRa, Tokyo, Japan). The quantitative real-time PCR was performed using the SYBR ${ }^{\circledR}$ Premix Ex Taq ${ }^{\mathrm{TM}}$ II reagent kit (TaKaRa) and specific primers on a CFX96 real-time PCR system (Bio-Rad, USA). The PCR amplification conditions were $95^{\circ} \mathrm{C}$ for $5 \mathrm{~min}, 95^{\circ} \mathrm{C}$ for $10 \mathrm{~s}, 55^{\circ} \mathrm{C}$ for $30 \mathrm{~s}$, and $72^{\circ} \mathrm{C}$ for $10 \mathrm{~s}$, for 40 cycles. The primers were synthesized by Sangon
(Shanghai, China) and listed in Table 1. Gene expression was normalized to GAPDH as internal controls and calculated using the $2^{-\Delta \Delta \mathrm{Ct}}$ method.

2.13. Western Blotting. The renal tissues were homogenized in a cold RIPA lysis buffer (Thermo Fisher Scientific) including protease and phosphatase inhibitors (Sigma-Aldrich). Then, the total protein was extracted after being centrifuged at $12,000 \mathrm{rpm}$ for $15 \mathrm{~min}$ at $4^{\circ} \mathrm{C}$. The protein concentration was measured with the BCA method. After that, the protein was denatured by storing in the water of $100^{\circ} \mathrm{C}$ for $5 \mathrm{~min}$ after being mixed with the loading buffer, and the supernatant was used for western blotting analysis. Protein samples were separated by $10 \%$ serum dodecyl sulfate-polyacrylamide gels (SDSPAGE) and then transferred to polyvinylidene fluoride (PVDF) membranes (Merck Millipore, USA). The membranes were blocked with $5 \%$ nonfat milk and incubated with primary antibodies against MMP-9 (1:2000, ab76003, Abcam, Cambridge, UK), $\alpha$-SMA ( $1: 1000$, AF1032, Affinity Biosciences, USA), ICAM-1 ( $1: 2000$, BF0097, Affinity Biosciences, USA), TGF$\beta 1$ (1:1000, ab215715, Abcam), Smad7 (1:1000, ab216428, Abcam), HIF1A (1:2000, BF0593, Affinity Biosciences, USA), total Smad3 (1:1000, ab40854, Abcam), p-Smad3 (1:2000, ab52903, Abcam), GAPDH (1:5000, D190090, Sangon, Shanghai, China), and $\beta$-actin $(1: 5000$, ab8227, Abcam) for a whole night at $4^{\circ} \mathrm{C}$, followed by incubation with goat antirabbit antibody (ab205718, 1:5000) and goat anti-mouse (ab205719, 1:5000) for $1 \mathrm{~h}$ at room temperature. GAPDH was used as an internal control. The blots were visualized with an ECL kit (P0018S, Beyotime, Beijing, China), and the protein intensity was quantified with ImageJ software.

2.14. Statistical Analysis. All the data were presented as mean \pm standard deviation (SD). Statistical analysis was performed by SPSS software (version 21.0; SPSS Inc., Chicago, IL, USA). Student's $t$-test was applied to the analysis of two groups, and a comparison between multiple groups was done using one-way ANOVA. $P<0.05$ is considered statistically significant.

\section{Results}

3.1. Identification of Absorbable Components in XXD Aqueous Extract. In this study, the phytochemical compositions of XXD were identified using UHPLC-Q-TOF-MS analysis based on the TCM MS/MS Library in SCIEX OS software. The base peak chromatograms of the XXD aqueous extract in positive and negative ion modes are presented in Figure 1. The identification results for the constituents in XXD are presented in Table S1 and Table S2. Fifty-five compounds were identified under the positive ion mode and sixty-seven compounds were identified under the negative ion mode. Using the Molinspiration to calculate the identified compounds of XXD, we obtained absorption parameters that could estimate whether the chemical compositions could be absorbed. As a result, Table 2 indicated that there were a total of 58 components that met the five principles of drug absorption after eliminating the redundancy. 
TABle 1: Primers used for qRT-PCR.

\begin{tabular}{|c|c|c|}
\hline Gene name & Forward $\left(5^{\prime} \sim 3^{\prime}\right)$ & Reverse $\left(5^{\prime} \sim 3^{\prime}\right)$ \\
\hline TNF- $\alpha$ & CAGGCGGTGCCTATGTCTC & CGATCACCCCGAAGTTCAGTAG \\
\hline IL-6 & GGCGGATCGGATGTTGTGAT & GGACCCCAGACAATCGGTTG \\
\hline IL- $1 \beta$ & GAAATGCCACCTTTTGACAGTG & TGGATGCTCTCATCAGGACAG \\
\hline MCP-1 & CTGAGTTGACTCCTACTGTGGA & TCTTCCCAGGGTCGATAAAGT \\
\hline Collagen-1 & AATGGAAGTTCTACTCGCGTAGG & TTCTCGCCTGGTTGACCTTTG \\
\hline TP53 & ACCTATGGAAACTACTTCCTGAAA & ACCATCGCTATCTGAGCAGC \\
\hline VEGFA & GGCAAAAACGAAAGCGCAAG & ATTAGACAGCAGCGGGCAC \\
\hline PTGS2 & GTTCCACCCGCAGTACAGAA & AGGGCTTCAGCATAAAGCGT \\
\hline TLR4 & ATCCCCTGAGGCATTTAGGC & GTCAAATTGCACAGGCCCTC \\
\hline NOS3 & GACCCACTGGTGTCCTCTTG & CTCCGTTTGGGGCTGAAGAT \\
\hline EGFR & ACGAGTAACAAGCTCACGCA & GATCACACTTTTGGCCCTGT \\
\hline PPARG & ACTTTGGGATCAGCTCCGTG & TGGGCGGTTGATTTGTCTGT \\
\hline HIF1A & CCAATGTCGGAGTTTGGAAAACA & GTGGCAACTGATGAGCAAGC \\
\hline HMOX1 & GTGCCACCAAGTTCAAGCAG & CAGCTCCTGCAACTCCTCAA \\
\hline GAPDH & TGTCAAGCTCATTTCCTGGTATG & TTATGGGGGTCTGGGATGGA \\
\hline
\end{tabular}

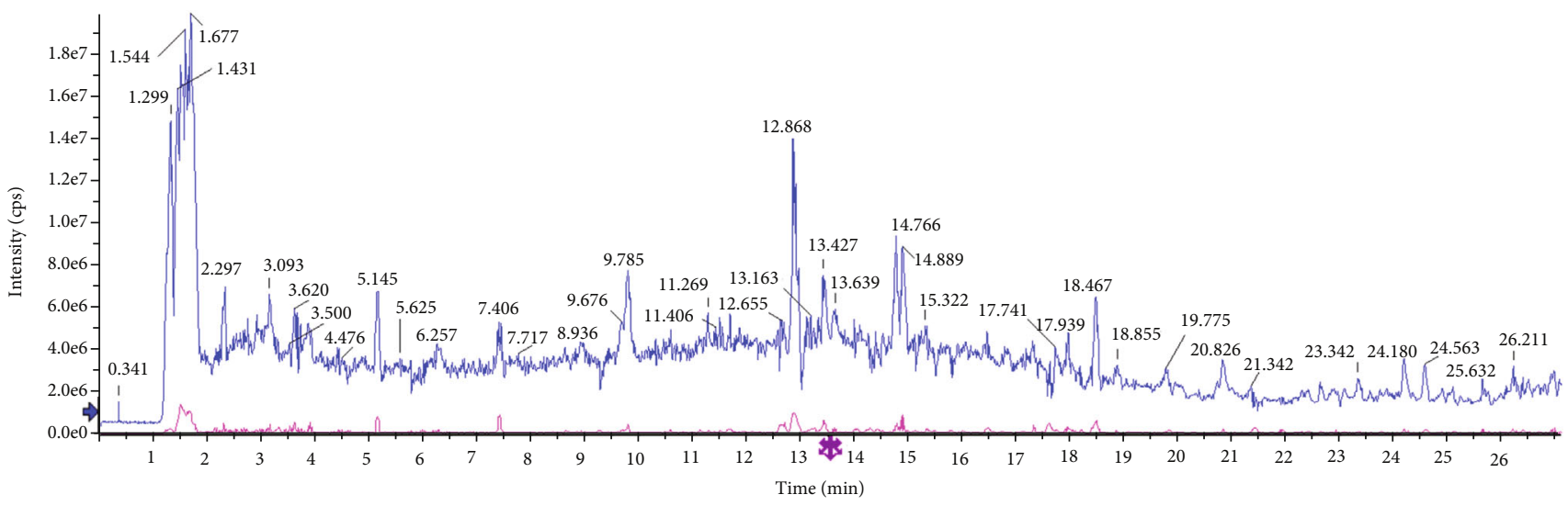

O IDA Survey from Xiaoyu Xiezhou Drink POS.wiff2 (sample 1)-Xiaoyu Xiezhou Drink_48 mg ml_POS_Experiment 1, +IDA TOF MS (50-1500)

O IDA Dependent Sum from Xiaoyu Xiezhou Drink POS. wiff2 (sample 1)-Xiaoyu Xiezhou Drink_48 mg ml_POS_Experiment 2-13, +IDA TOF MSMS (25-1500)

(a)

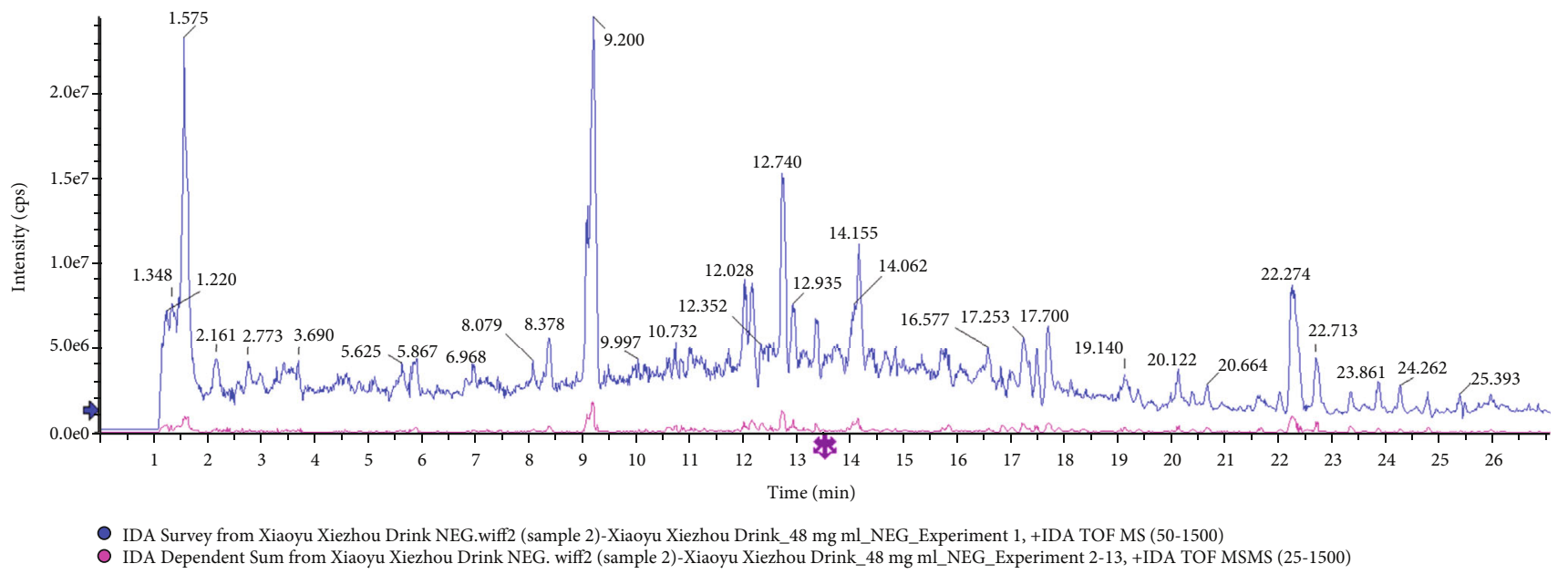

(b)

FIgURE 1: The base peak chromatograms of the XXD extract by UPLC-Q-TOF-MS analysis in positive (a) and negative (b) ion modes. 
TABLE 2: The absorbable components of XXD aqueous extract.

\begin{tabular}{|c|c|c|c|c|c|c|c|}
\hline No. & Component name & Formula & Mass & $\mathrm{nON}$ & nOHNH & miLogP & Results \\
\hline 1 & Betaine & $\mathrm{C} 5 \mathrm{H} 11 \mathrm{NO} 2$ & 118.0861 & 3 & 0 & -5.41 & $\checkmark$ \\
\hline 2 & Trigonelline & C7H7NO2 & 138.0551 & 3 & 0 & -5.4 & $\checkmark$ \\
\hline 3 & Proline & C5H9NO2 & 116.0706 & 3 & 2 & -1.72 & $\checkmark$ \\
\hline 4 & Stachydrine & C7H13NO2 & 144.1018 & 3 & 0 & -5.31 & $\checkmark$ \\
\hline 5 & Adenine & C5H5N5 & 136.0616 & 5 & 3 & 0.23 & $\checkmark$ \\
\hline 6 & Cytidine & $\mathrm{C} 9 \mathrm{H} 13 \mathrm{~N} 3 \mathrm{O} 5$ & 244.093 & 8 & 5 & -1.93 & $\checkmark$ \\
\hline 7 & Nicotinic acid & C6H5NO2 & 124.0391 & 3 & 1 & 0.27 & $\checkmark$ \\
\hline 8 & Nicotinamide & $\mathrm{C} 6 \mathrm{H} 6 \mathrm{~N} 2 \mathrm{O}$ & 123.0552 & 3 & 2 & -0.48 & $\checkmark$ \\
\hline 9 & Adenosine & $\mathrm{C} 10 \mathrm{H} 13 \mathrm{~N} 5 \mathrm{O} 4$ & 268.1038 & 9 & 5 & -0.85 & $\checkmark$ \\
\hline 10 & 6-Hydroxypurine & C5H4N4O & 137.0457 & 5 & 2 & -0.73 & $\checkmark$ \\
\hline 11 & Phenylalanine & C9H11NO2 & 166.0862 & 3 & 3 & -1.23 & $\checkmark$ \\
\hline 12 & Higenamine & $\mathrm{C} 16 \mathrm{H} 17 \mathrm{NO} 3$ & 272.1284 & 4 & 4 & 2 & $\checkmark$ \\
\hline 13 & 4-Hydroxybenzoic acid & $\mathrm{C} 7 \mathrm{H} 6 \mathrm{O} 3$ & 139.0389 & 3 & 2 & 1.37 & $\checkmark$ \\
\hline 14 & Catechin & C15H14O6 & 291.0866 & 6 & 5 & 1.37 & $\checkmark$ \\
\hline 15 & Epicatechin & $\mathrm{C} 15 \mathrm{H} 14 \mathrm{O} 6$ & 291.0866 & 6 & 5 & 1.37 & $\checkmark$ \\
\hline 16 & Daphnetin & С9H6O4 & 179.0339 & 4 & 2 & 1.25 & $\checkmark$ \\
\hline 17 & Vitamin B2 & C17H20N4O6 & 377.1458 & 10 & 5 & -0.76 & $\checkmark$ \\
\hline 18 & Geniposide & C17H24O10.NH3 & 406.1711 & 10 & 5 & -1.53 & $\checkmark$ \\
\hline 19 & Syringaldehyde & C9H10O4 & 183.0652 & 4 & 1 & 1.08 & $\checkmark$ \\
\hline 20 & Calycosin-7-O-glucoside & $\mathrm{C} 22 \mathrm{H} 22 \mathrm{O} 10$ & 447.1284 & 10 & 5 & 0.59 & $\checkmark$ \\
\hline 21 & Glycitin & $\mathrm{C} 22 \mathrm{H} 22 \mathrm{O} 10$ & 447.1284 & 10 & 5 & 0.36 & $\checkmark$ \\
\hline 22 & Scutellarein & $\mathrm{C} 15 \mathrm{H} 10 \mathrm{O} 6$ & 287.0553 & 6 & 4 & 2.2 & $\checkmark$ \\
\hline 23 & Liquiritin & $\mathrm{C} 21 \mathrm{H} 22 \mathrm{O} 9$ & 419.1337 & 9 & 5 & 0.41 & $\checkmark$ \\
\hline 24 & Ononin & $\mathrm{C} 22 \mathrm{H} 22 \mathrm{O} 9$ & 431.1337 & 9 & 4 & 1.31 & $\checkmark$ \\
\hline 25 & 3-Hydroxy-9,10-dimethoxypterocarpan & C17H16O5 & 301.107 & 5 & 1 & 2.55 & $\checkmark$ \\
\hline 26 & Calycosin & $\mathrm{C} 16 \mathrm{H} 12 \mathrm{O} 5$ & 285.0755 & 5 & 2 & 2.38 & $\checkmark$ \\
\hline 27 & Isomucronulatol & $\mathrm{C} 17 \mathrm{H} 18 \mathrm{O} 5$ & 303.1228 & 5 & 2 & 2.63 & $\checkmark$ \\
\hline 28 & Isomucronulatol-7-O-glucoside & $\mathrm{C} 23 \mathrm{H} 28 \mathrm{O} 10$ & 465.1762 & 10 & 5 & 0.84 & $\checkmark$ \\
\hline 29 & Nodakenin & $\mathrm{C} 20 \mathrm{H} 24 \mathrm{O} 9$ & 409.1497 & 9 & 4 & 0.47 & $\checkmark$ \\
\hline 30 & Emodin & $\mathrm{C} 15 \mathrm{H} 10 \mathrm{O} 5$ & 271.06 & 5 & 3 & 3.01 & $\checkmark$ \\
\hline 31 & Chrysophanol & $\mathrm{C} 15 \mathrm{H} 10 \mathrm{O} 4$ & 255.065 & 4 & 2 & 3.54 & $\checkmark$ \\
\hline 32 & Cinnamic acid & $\mathrm{C} 9 \mathrm{H} 8 \mathrm{O} 2$ & 149.0596 & 2 & 1 & 1.91 & $\checkmark$ \\
\hline 33 & Naringenin & $\mathrm{C} 15 \mathrm{H} 12 \mathrm{O} 5$ & 273.0758 & 5 & 3 & 2.12 & $\checkmark$ \\
\hline 34 & Diosmetin & $\mathrm{C} 16 \mathrm{H} 12 \mathrm{O} 6$ & 301.0708 & 6 & 3 & 2.28 & $\checkmark$ \\
\hline 35 & Formononetin & $\mathrm{C} 16 \mathrm{H} 12 \mathrm{O} 4$ & 269.0808 & 4 & 1 & 3.1 & $\checkmark$ \\
\hline 36 & Histidine & $\mathrm{C} 6 \mathrm{H} 9 \mathrm{~N} 3 \mathrm{O} 2$ & 154.0623 & 5 & 4 & -3 & $\checkmark$ \\
\hline 37 & Glutamic acid & C5H9NO4 & 146.0458 & 5 & 4 & -3.25 & $\checkmark$ \\
\hline 38 & D-(+)-Mannose & $\mathrm{C} 6 \mathrm{H} 12 \mathrm{O} 6$ & 179.056 & 6 & 5 & -2.64 & $\checkmark$ \\
\hline 39 & Citric acid & $\mathrm{C} 6 \mathrm{H} 8 \mathrm{O} 7$ & 191.02 & 7 & 4 & -1.98 & $\checkmark$ \\
\hline 40 & Amber acid & $\mathrm{C} 4 \mathrm{H} 6 \mathrm{O} 4$ & 117.0194 & 4 & 2 & -0.66 & $\checkmark$ \\
\hline 41 & Isoleucine & C6H13NO2 & 130.0874 & 3 & 3 & -1.41 & $\checkmark$ \\
\hline 42 & Gallic acid & C7H6O5 & 169.0144 & 5 & 4 & 0.59 & $\checkmark$ \\
\hline 43 & Protocatechuic acid & $\mathrm{C} 7 \mathrm{H} 6 \mathrm{O} 4$ & 153.0193 & 4 & 3 & 0.88 & $\checkmark$ \\
\hline 44 & Hydroxytyrosol & C8H10O3 & 153.0558 & 3 & 3 & 0.52 & $\checkmark$ \\
\hline 45 & L-Tryptophan & $\mathrm{C} 11 \mathrm{H} 12 \mathrm{~N} 2 \mathrm{O} 2$ & 203.0829 & 4 & 4 & -1.08 & $\checkmark$ \\
\hline 46 & Esculetin & C9H6O4 & 177.0196 & 4 & 2 & 1.02 & $\checkmark$ \\
\hline 47 & Caffeic acid & $\mathrm{C} 9 \mathrm{H} 8 \mathrm{O} 4$ & 179.0351 & 4 & 3 & 0.94 & $\checkmark$ \\
\hline 48 & 7-Hydroxycoumarin & $\mathrm{C} 9 \mathrm{H} 6 \mathrm{O} 3$ & 161.0244 & 3 & 1 & 1.51 & $\checkmark$ \\
\hline
\end{tabular}


TABle 2: Continued.

\begin{tabular}{|c|c|c|c|c|c|c|c|}
\hline No. & Component name & Formula & Mass & $\mathrm{nON}$ & nOHNH & miLogP & Results \\
\hline 49 & p-Coumaric acid & $\mathrm{C} 9 \mathrm{H} 8 \mathrm{O} 3$ & 163.0403 & 3 & 2 & 1.43 & $\checkmark$ \\
\hline 50 & Isoferulic acid & $\mathrm{C} 10 \mathrm{H} 10 \mathrm{O} 4$ & 193.051 & 4 & 2 & 1.25 & $\checkmark$ \\
\hline 51 & Rosmarinic acid & $\mathrm{C} 18 \mathrm{H} 16 \mathrm{O} 8$ & 359.0776 & 8 & 5 & 1.63 & $\checkmark$ \\
\hline 52 & Eriodictyol & $\mathrm{C} 15 \mathrm{H} 12 \mathrm{O} 6$ & 287.0563 & 6 & 4 & 1.63 & $\checkmark$ \\
\hline 53 & Luteolin & $\mathrm{C} 15 \mathrm{H} 10 \mathrm{O} 6$ & 285.0408 & 6 & 4 & 1.97 & $\checkmark$ \\
\hline 54 & Quercetin & $\mathrm{C} 15 \mathrm{H} 10 \mathrm{O} 7$ & 301.0357 & 7 & 5 & 1.68 & $\checkmark$ \\
\hline 55 & Vanillic acid & $\mathrm{C} 8 \mathrm{H} 8 \mathrm{O} 4$ & 167.0352 & 4 & 2 & 1.19 & $\checkmark$ \\
\hline 56 & Aurantio-obtusin & $\mathrm{C} 17 \mathrm{H} 14 \mathrm{O} 7$ & 329.0669 & 7 & 3 & 3.01 & $\checkmark$ \\
\hline 57 & Rhein & $\mathrm{C} 15 \mathrm{H} 8 \mathrm{O} 6$ & 283.0251 & 6 & 3 & 3 & $\checkmark$ \\
\hline 58 & Asiatic acid & $\mathrm{C} 30 \mathrm{H} 48 \mathrm{O} 5$ & 487.3433 & 5 & 4 & 4.7 & $\checkmark$ \\
\hline
\end{tabular}

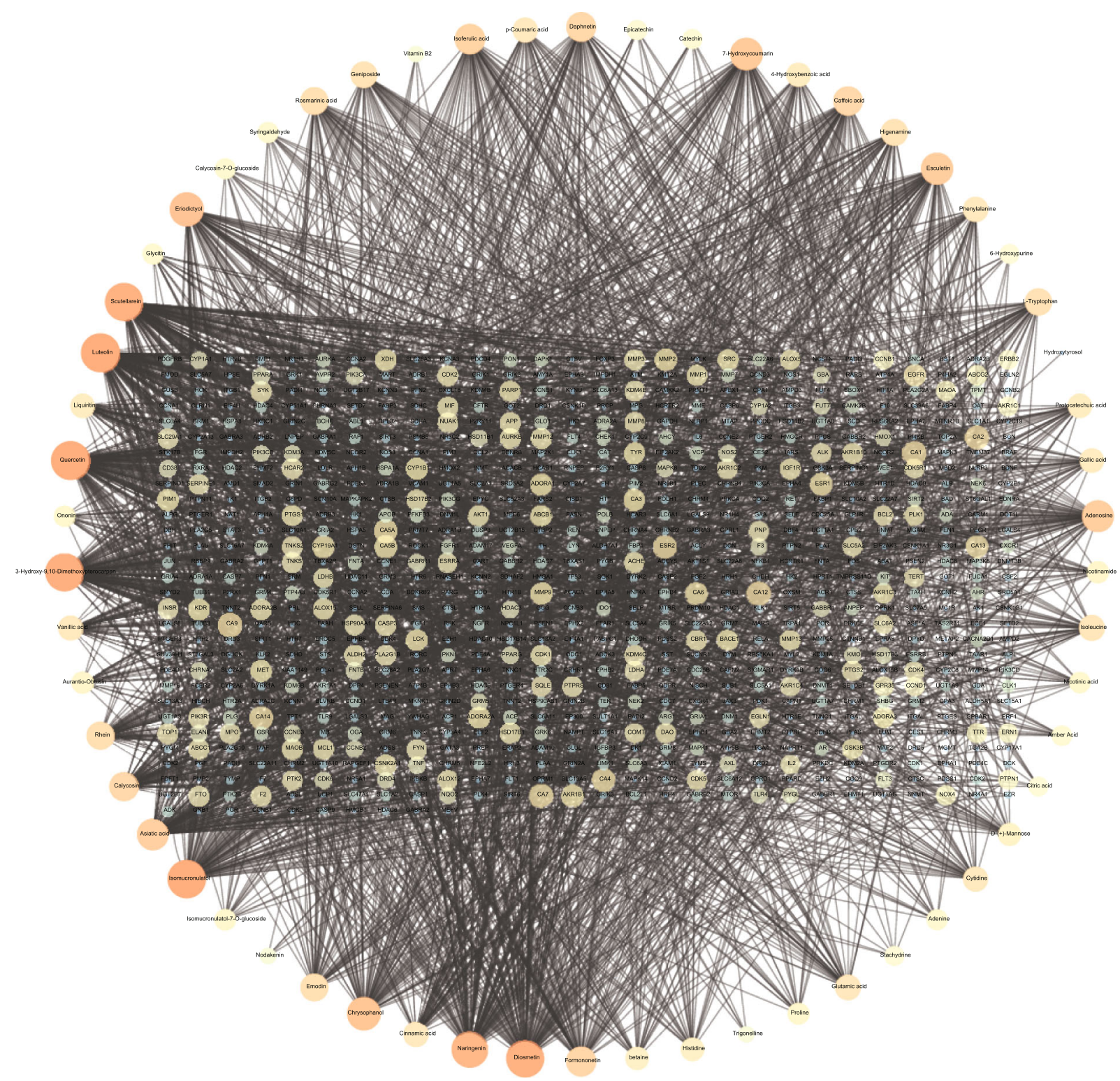

Figure 2: The compound-target network for XXD. The nodes in circles represent candidate absorbable compounds, and nodes with the rectangular arrangement represent potential protein targets. The edges represent the interactions between them. Node size and color are visualized by using their degree. 


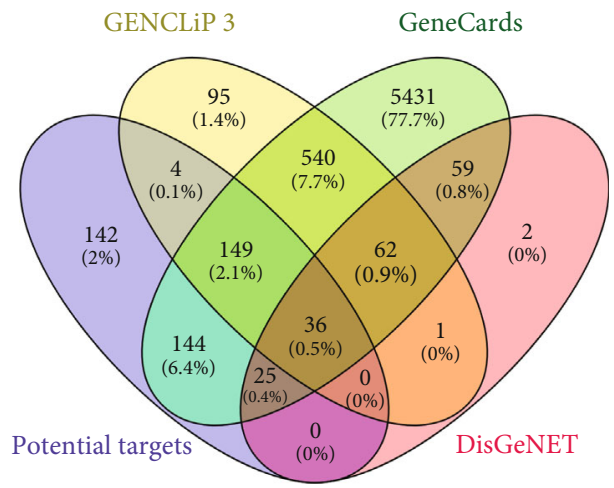

(a)

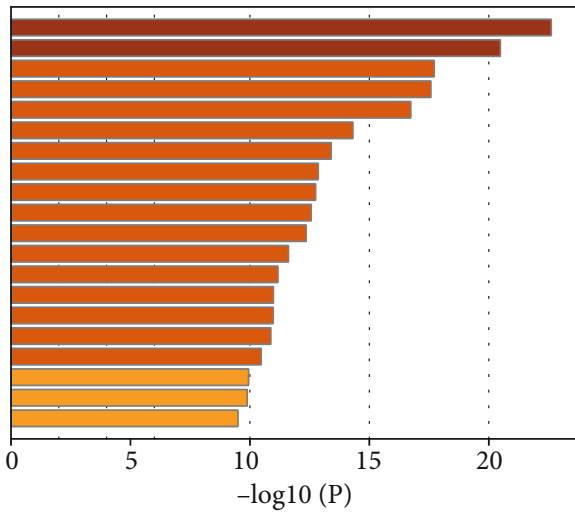

GO-0006979: response to oxidative stres GO:0072593: reactive oxygen species metabolic process GO:0071496; cellular response to external stimats hsa04066: HE-1 sian response to GO-0070482: response to oxygen levels hsa05200: Pathways in cancer GO:0008015: blood circulation ko05418: Fluid shear stress and atheroscleros GO:2001234: negative regulation of apoptotic signaling pathway GO:0062013: positive regulation of small molecule metabolic process GO:0035690: cellular response to drug GO:0002366: leukocyte activation involved in immune response hsa05205: Proteoglycans in cancer GO:0009266: response to temperature stimulus GO:0010638: positive regulation of organelle organization GO:0010506: regulation of autophagy ncription from RNA polymerase II promoter in response to hypox hsa05167: kaposi sarcoma-associated herpesvirus infection GO:1901214: regulation of neuron deat GO-0040008: regulation of growth

(b)

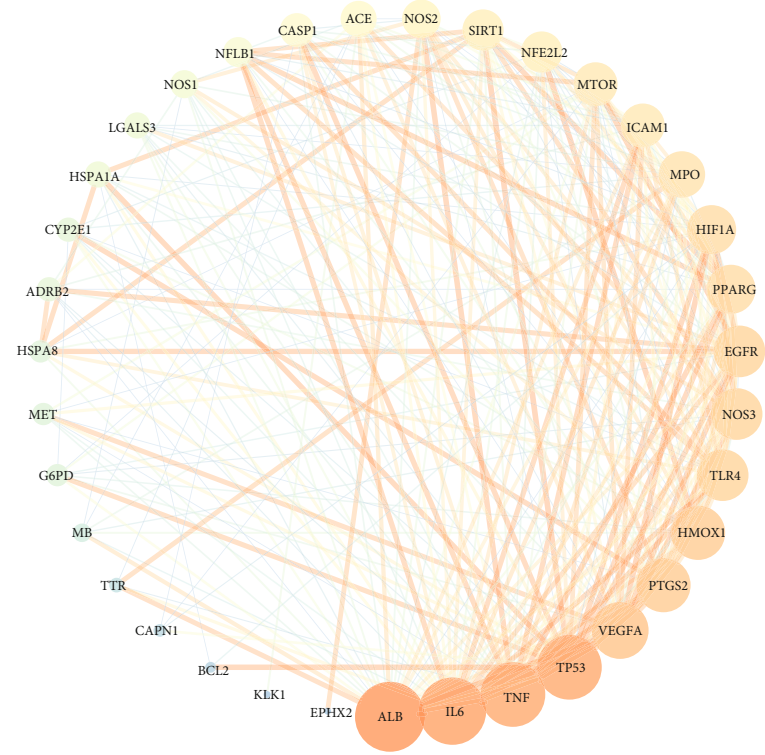

(c)

FIgURE 3: Target identification of XXD on AKI and analysis. (a) The Venn diagram of the targets in both XXD targets and AKI targets. (b) The GO-BP and KEGG pathway enrichment analyses of 36 involved targets in XXD. (c) The PPI network analysis of 35 potential targets. Node size and color are proportional to the target degree in the network.

3.2. Absorbable Component-Target Network Analysis. Among the 58 candidate bioactive components, 2622 protein targets were retrieved from the SwissTargetPrediction and STITCH databases. After eliminating the overlaps, 800 drug-protein targets of XXD were obtained for further analyses. As shown in Figure 2, There are 858 nodes (800 compound target nodes and 58 compound nodes) and 2620 edges which composed the absorbable component-target network. In this network, we found some special absorbable compounds with a higher degree interacting with multiple targets such as luteolin (degree $=114$ ), quercetin $($ degree $=114)$, isomucronulatol $($ degree $=111)$, 3-hydroxy-9,10dimethoxypterocarpan $($ degree $=110)$, scutellarein $($ degree $=109$ 


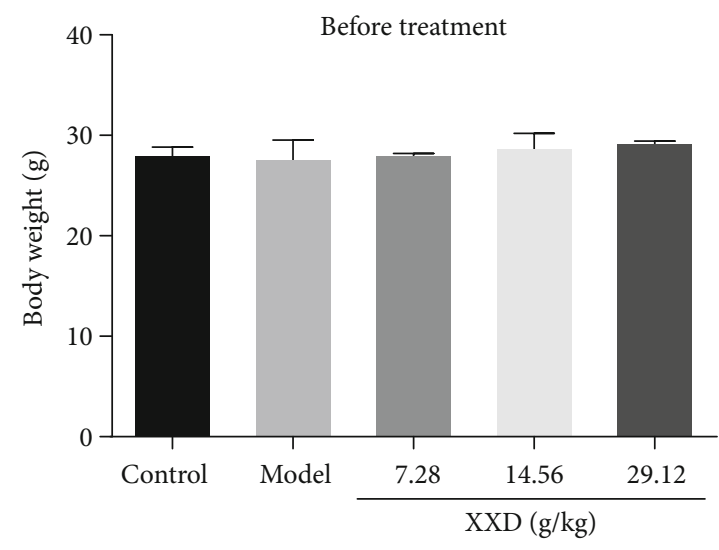

(a)

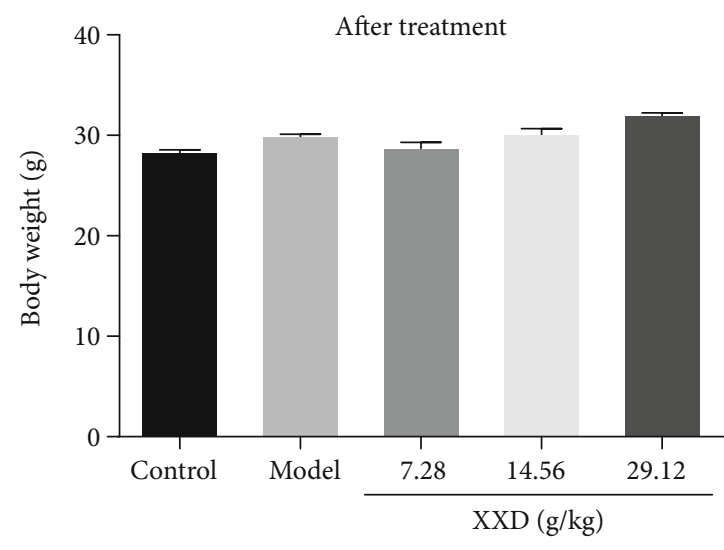

(b)

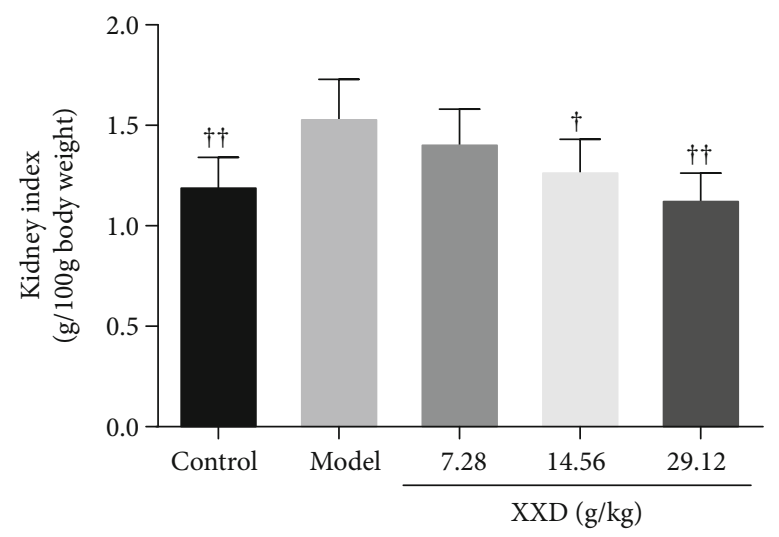

(c)

FIGURE 4: Effect of XXD on body weight of AKI mice. (a) The body weight of mice before XXD treatment. (b) The body weight of mice after XXD treatment. (d) The kidney index was calculated via kidney weight/body weight. $n=10$ mice per group. All data are expressed as means \pm SD. ${ }^{\boldsymbol{\Delta}} P<0.05,{ }^{\boldsymbol{\Lambda}} P<0.01$ vs. the model group.

), diosmetin (degree $=109)$, and naringenin (degree $=102)$. These high-degree absorbable components in the network may account for the essential therapeutic effects of XXD on AKI.

3.3. Target Identification and Analysis. In total, 887 AKIrelated genes were collected from the GenCLip 3 database. 6746 AKI-related genes were collected from the GeneCards database. And 185 AKI-related genes were acquired based on the DisGeNET database. Then, drug targets of XXD were mapped with AKI-related genes. As a result, 36 crossover genes of 58 components in XXD were associated with AKI and are shown in Figure 3(a) such as TLR4, ACE, HSPA8, SIRT1, EGFR, HSPA1A, HMOX1, NOS2, NOS1, NOS3, ADRB2, PTGS2, MET, IL-6, CYP2E1, LGALS3, EPHX2, TTR, TNF, SLC22A12, MPO, MTOR, VEGFA, TP53, BCL2, CAPN1, PPARG, KLK1, MB, ALB, CASP1, ICAM1, NFE2L2, NFKB1, HIF1A, and G6PD.

To illustrate the biological characteristics of AKI-related genes of XXD on AKI, we performed GO and KEGG pathway enrichment analyses of the 36 involved targets via the Metascape database. The detailed GO terms and pathway information of XXD on AKI are shown in Figure 3(b). As a result, the targets of XXD were enriched in response to oxidative stress (GO:0006979), reactive oxygen species metabolic process (GO: 0072593), and cellular response to external stimulus (GO:
0071496), etc. Meanwhile, we observed that the HIF1 signaling pathway (hsa04066), proteoglycans in cancer (hsa05205), and Kaposi sarcoma-associated herpesvirus infection (hsa05167) were mainly involved with the 36 targets of 58 components in XXD. Based on those GO and KEGG pathway enrichment analyses, it is possible that the anti-AKI effect of XXD results from a complex multibiological process and multipathway synergetic effect.

To study the interaction of the 36 targets in vivo and search for the hub genes, PPI network analysis was carried out. There were 35 nodes (SLC22A12, the discrete node, was removed) and 273 edges in total. The topological feature analysis of the PPI is based on the major parameters of "degree." In this network, we used the median value of node degree (20) as a cutoff point primarily. Eventually, 12 major hubs were picked out as hub genes for further study. These genes were ALB (degree=29), IL-6 (degree=28), TNF $($ degree $=27)$, TP53 $($ degree $=27)$, VEGFA $($ degree $=24)$, PTGS2 $\quad($ degree $=23), \quad$ HMOX1 $\quad($ degree $=23), \quad$ NOS3 $($ degree $=22), \quad$ TLR4 $\quad($ degree $=22), \quad$ EGFR $\quad($ degree $=22)$, PPARG (degree $=21)$, and HIF1A (degree = 21) $($ Figure 3(c)).

3.4. XXD Ameliorated Kidney Function in AKI Mice. To investigate the effect of XXD on aging mice with AKI, liver and kidney function indicators were evaluated. As shown 


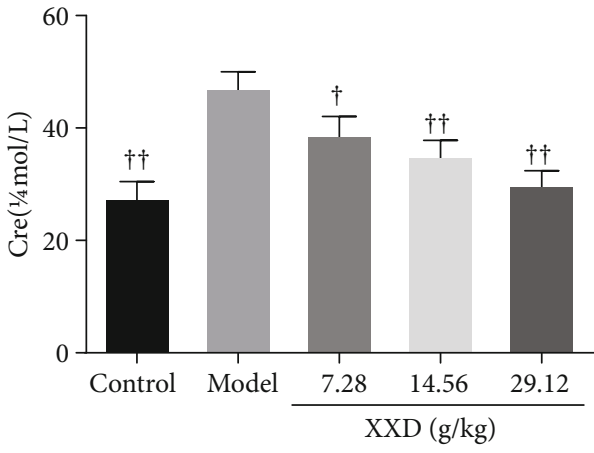

(a)

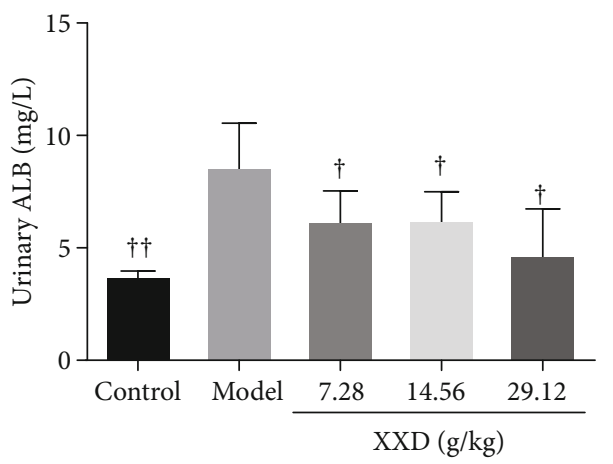

(c)

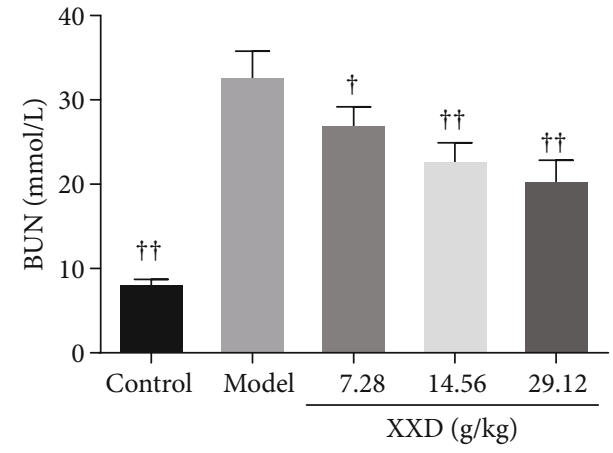

(b)

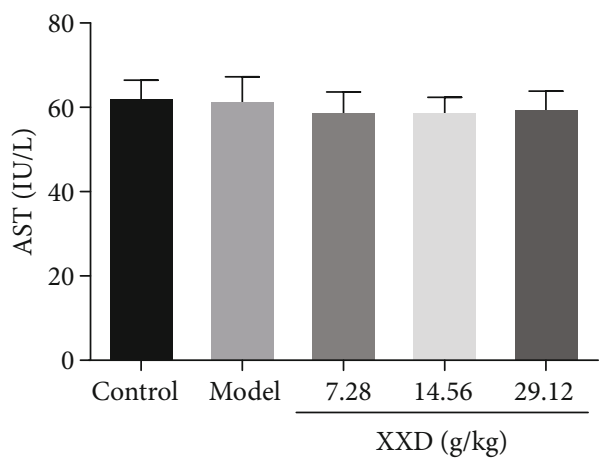

(d)

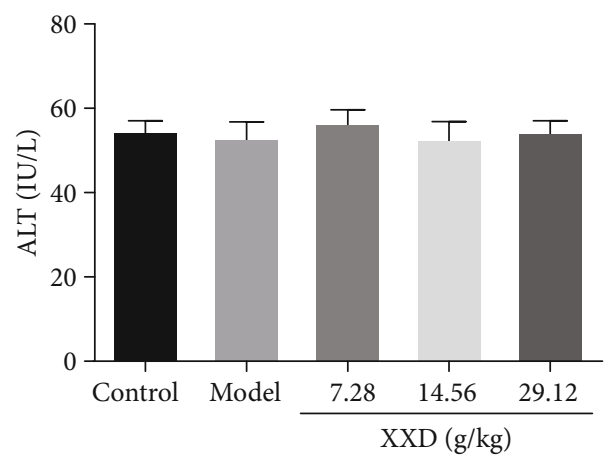

(e)

FIGURE 5: Effect of XXD on liver and kidney function of AKI mice: (a) serum creatinine (Cre), (b) blood urea nitrogen (BUN), (c) urinary albumin (ALB), (d) serum aspartate aminotransferase (AST), and (e) alanine aminotransferase (ALT). $n=10$ mice per group. All data are expressed as means \pm SD. ${ }^{\boldsymbol{\Delta}} P<0.05,{ }^{\boldsymbol{\Delta}} P<0.01$ vs. the model group.

in Figures 4(a) and 4(b), the body weight of AKI mice has maintained a relatively stable status after XXD treatment. They did not differ significantly compared with the model group until 14 days. Compared with the control group, the kidney index in the model group was significantly increased; however, that level in the XXD groups (14.56 and $29.12 \mathrm{~g} / \mathrm{kg}$ ) was significantly lower than that in the model group (Figure 4(c)). Further, we found that treatment with XXD significantly lowered serum Cre (Figure 5(a)) and BUN (Figure 5(b)) levels, which were increased in the model group, in a relatively dose-dependent manner. Additionally, the urinary ALB level in the XXD-treated group was significantly lower than that in the model group (Figure 5(c)). Notably, there was no significant difference in AST (Figure 5(d)) and ALT (Figure 5(e)) levels in aging AKI mice treated with XXD.
3.5. XXD Alleviated I/R-Induced Kidney Injury of Aging Mice. The kidney tissues were collected and stained with $\mathrm{H} \& \mathrm{E}$ and Masson to explore the histopathological changes and the degree of renal fibrosis, respectively. As shown in Figure 6(a), the H\&E staining results showed that the renal tissue structure was tightly arranged, the structure of the glomerulus was clear, and the kidney tubules were complete and tightly packed in the control group. In contrast to the control group, the glomerular basement membranes of the glomerular were significantly thickened, cells around the glomerulus were disordered accompanied with glomerular hypertrophy, and the kidney tubules exhibited diffuse expansion. These pathological changes were improved under XXD treatment, compared with those in the model group. Masson staining showed aging mice with AKI revealed moderate renal interstitial fibrosis. The collagen fibers in the 

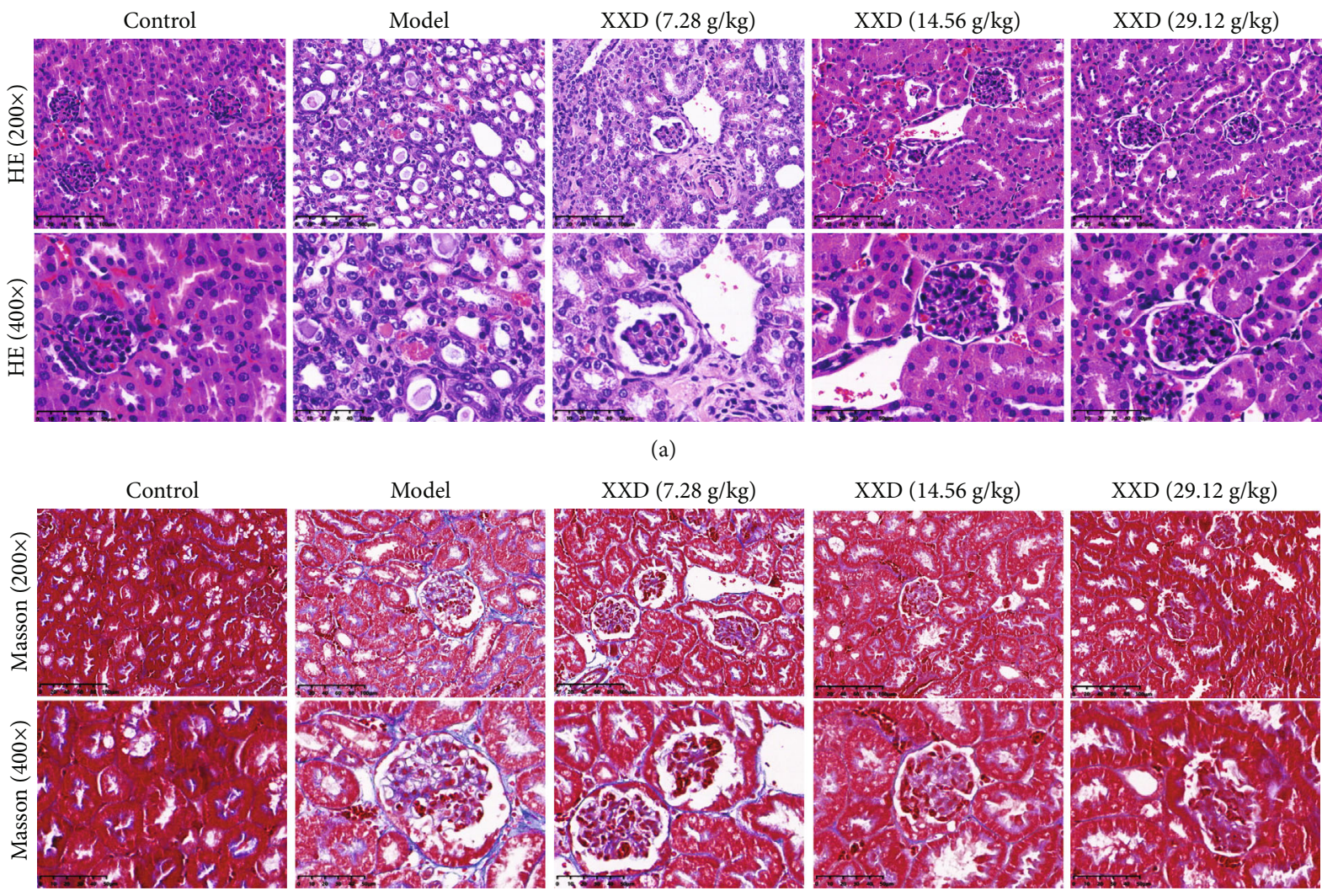

(b)
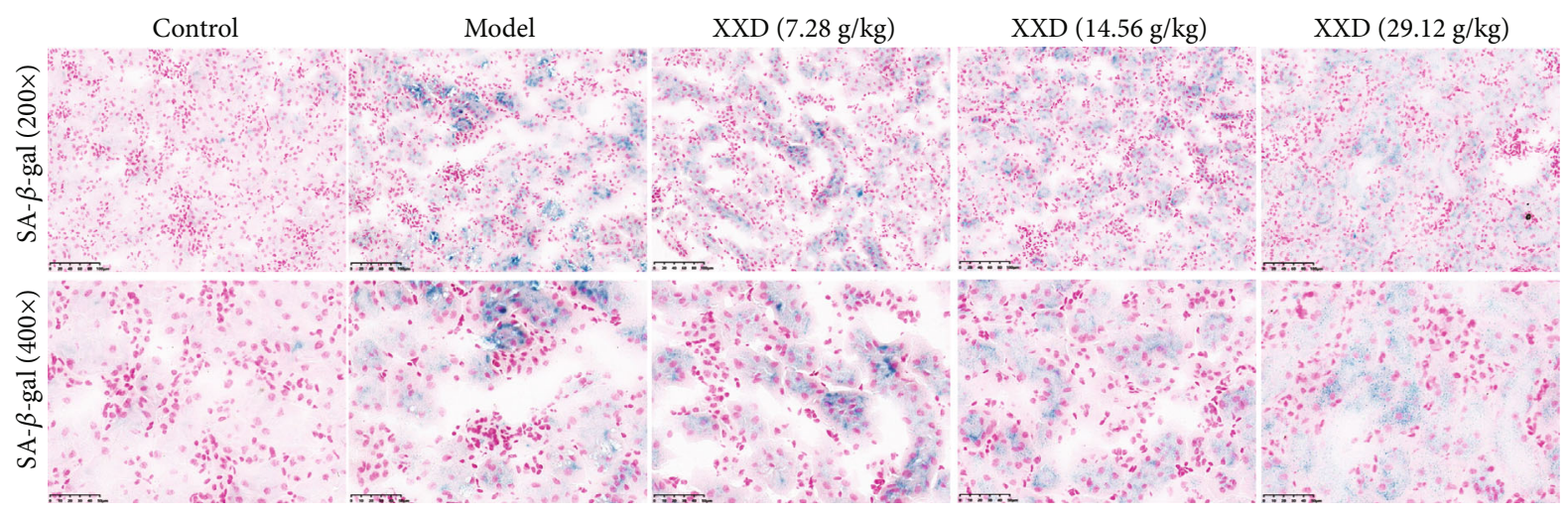

(c)

FIGURE 6: Effect of XXD on renal histopathology of the kidneys. (a) Hematoxylin and eosin (HE) and (b) Masson staining show changes in the control and model mice with XXD treatment. Bars represent $100 \mu \mathrm{m}$ and $50 \mu \mathrm{m}$. (c) SA- $\beta$-gal staining of kidney tissue in AKI mice. Semiquantitative analysis of SA- $\beta$-gal staining showed a significant decrease of SA- $\beta$-gal activity in the XXD-treated kidneys as compared with the model mice.

kidney tissue in each XXD-treated group, particularly at the XXD $(29.12 \mathrm{~g} / \mathrm{kg})$ group, were lower than that in the model group (Figure 6(b)). SA- $\beta$-gal is a common biomarker of senescence. In this study, the results showed that SA- $\beta$-gal activity was significantly lower in the XXD-treated kidneys than in the model groups (Figure 6(c)). Therefore, these results suggested that XXD could suppress the cellular senescence of kidney tissues in AKI aged mice.

3.6. XXD Suppressed Inflammation and Oxidative Stress in Kidney Tissue of AKI Mice. To elucidate the effect of the $\mathrm{XXD}$ on inflammation and oxidative stress in kidney tissue of AKI mice, we evaluated the expression levels of TNF- $\alpha$, IL-6, IL-1 $\beta$, MCP-1, MDA, SOD, and GSH. As shown in Figures $7(\mathrm{a})-7(\mathrm{~d}), \mathrm{XXD}$ significantly reduced the expression of TNF- $\alpha$, IL- 6 , IL- $1 \beta$, and MCP- 1 in the kidney tissue of AKI mice. Moreover, we also found that the mRNA levels of collagen-1 were significantly decreased in XXD-treated groups (Figure 7(e)). Interestingly, XXD markedly inhibited the expression of MDA (Figure 7(f)); also, SOD and GSH levels were also increased in the kidney tissue of AKI mice upon XXD treatment (Figures $7(\mathrm{~g})$ and $7(\mathrm{~h})$ ). These findings indicated the efficacy of XXD in protecting against AKI through inhibiting inflammation and oxidative stress. 


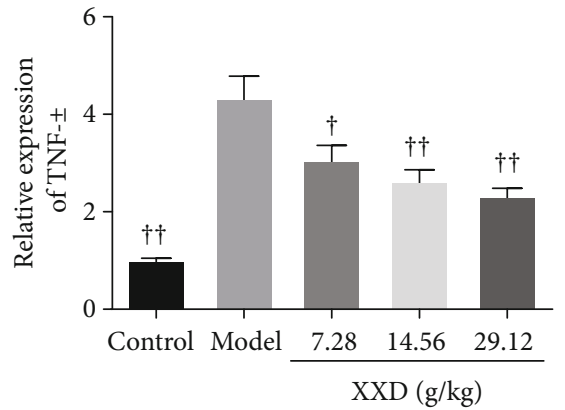

(a)

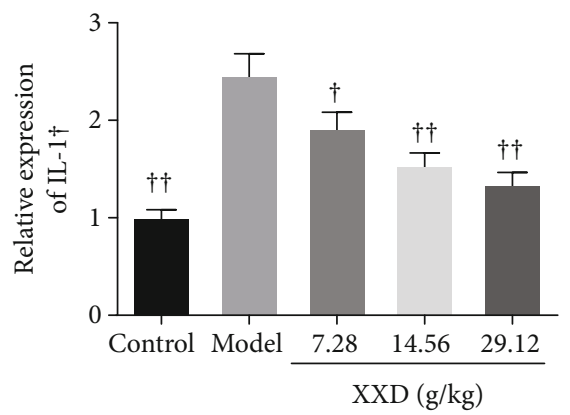

(c)

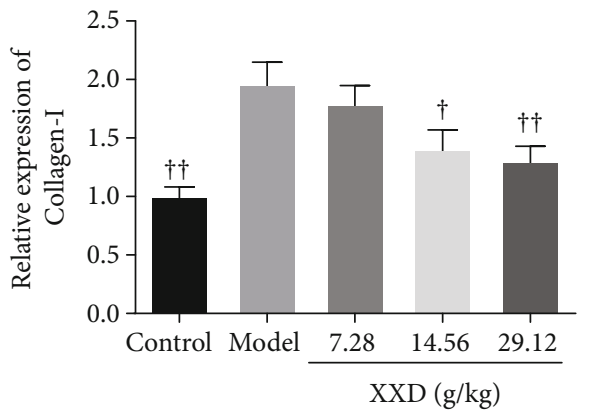

(e)

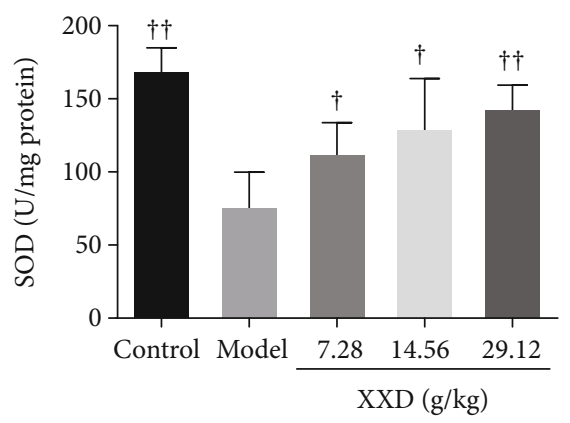

(g)

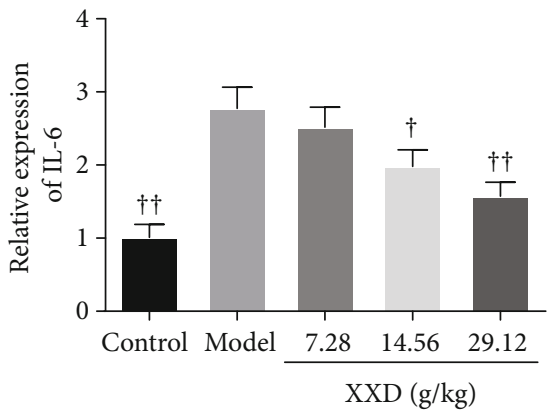

(b)

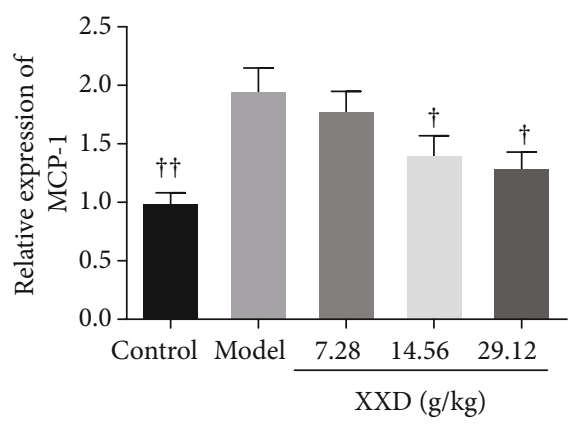

(d)

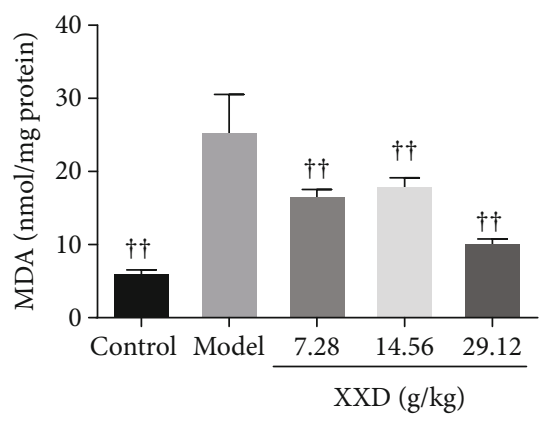

(f)

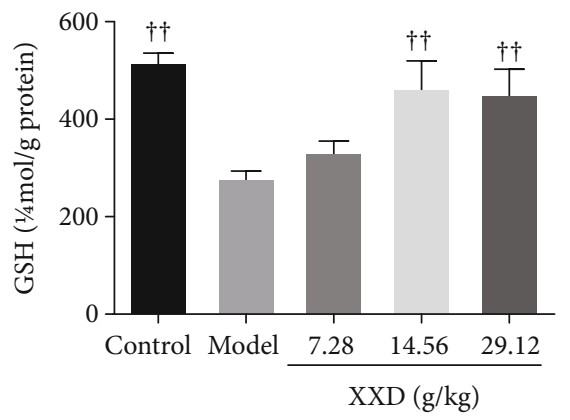

(h)

FIGURE 7: Effect of XXD on inflammation and oxidative stress in kidney tissue of AKI mice. The mRNA expression levels of (a) TNF- $\alpha$, (b) IL-6, (c) IL-1 $\beta$, (d) MCP-1, and (e) collagen-1 were measured using a qRT-PCR assay. The levels of (f) MDA, (g) SOD, and (h) GSH were detected using ELISA kits. Data are presented as the means $\pm \operatorname{SD}(n=10) .{ }^{\boldsymbol{\Delta}} P<0.05,{ }^{\boldsymbol{\Delta}} P<0.01$ vs. the model group.

3.7. XXD Downregulated $\alpha$-SMA, Collagen-1, and F4/80 and Upregulated p-H3 and Ki67 Expression in Mice with AKI. To further investigate the protective effect of XXD on the renal fibrosis of AKI mice, the expression levels of fibrotic factors, such as $\alpha$-SMA and collagen-1, are observed in Figures 8(a) and $8(\mathrm{~b})$. Compared with the control group, the expressions of $\alpha$-SMA and collagen- 1 were significantly increased in kid- neys of AKI mice. However, treatment with high-dose XXD effectively reversed the increase of $\alpha$-SMA and collagen-1. Furthermore, the presence in kidney tissue of the macrophage marker F4/80 intensively supports an inflammatory response to AKI after I/R injury. Compared to model mice, the expressions of F4/80 were significantly decreased after XXD treatment. To confirm the tubular repair induced by XXD, the 


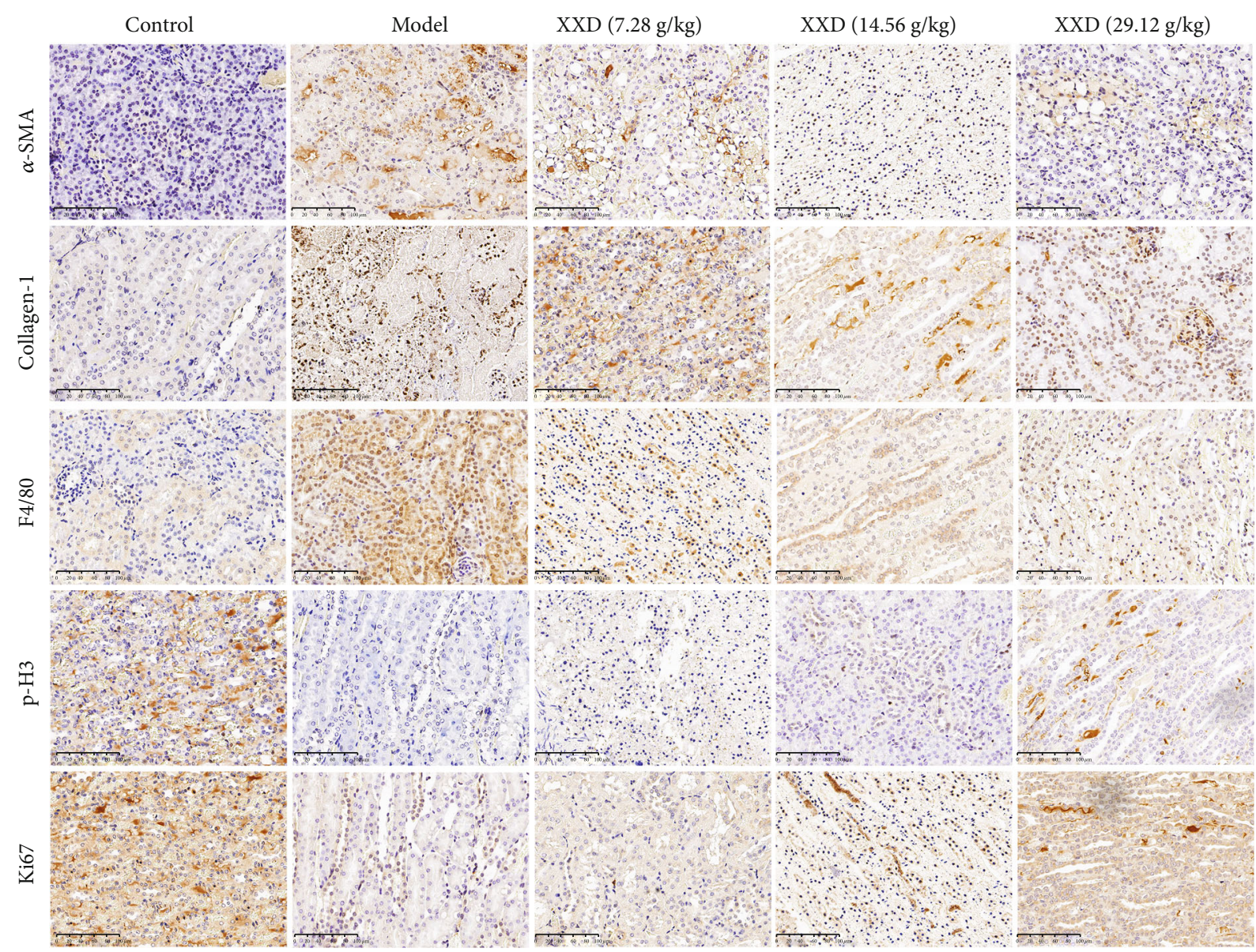

(a)

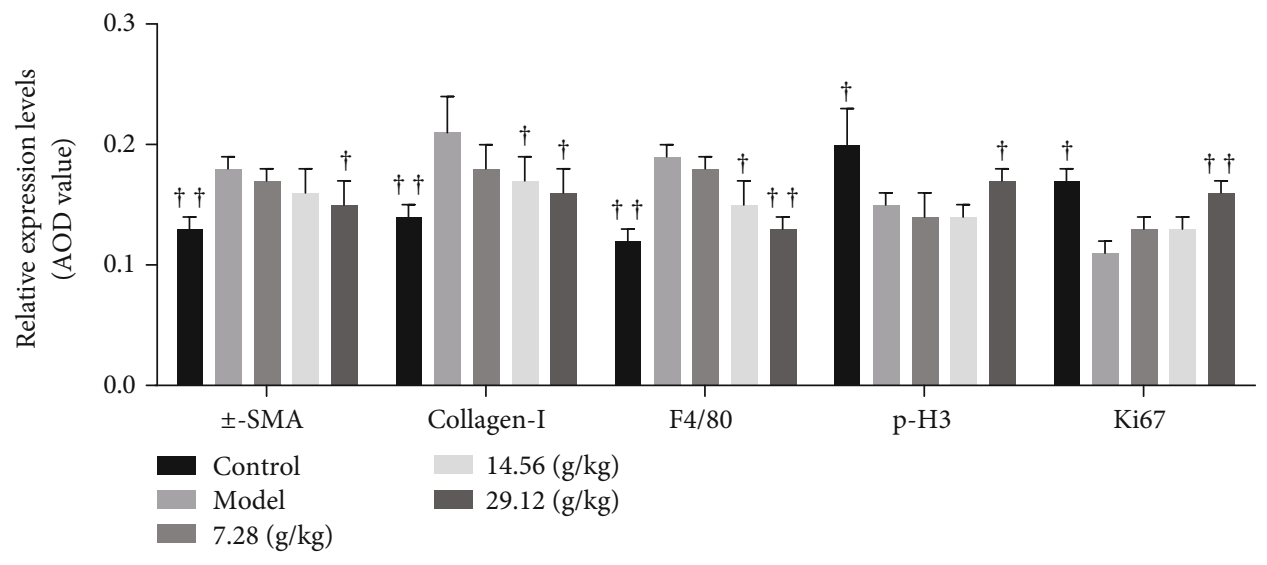

(b)

FIgURE 8: Effect of XXD on the renal fibrosis and tubular repair of AKI mice. (a) Representative immunohistochemical staining and (b) quantitative analysis of the expression of $\alpha$-SMA, collagen-1, F4/80, p-H3, and Ki67 in XXD groups as indicated. $n=10$ mice per group. All data are expressed as means $\pm \mathrm{SD} .{ }^{\boldsymbol{\Delta}} P<0.05,{ }^{\boldsymbol{\Delta}} P<0.01$ vs. the model group.

expression levels of p-H3 and Ki67 were also detected by using immunohistochemistry staining. The expression of $\mathrm{p}-\mathrm{H} 3$ was during the $\mathrm{M}$ phase of the cell cycle, and we found that the levels of $\mathrm{p}-\mathrm{H} 3$ were obviously increased in the high-dose XXD group compared with model mice. Also, the immunohistochemical staining demonstrated that the expression of Ki67, a well-accepted marker of cellular proliferation, was increased in the high-dose XXD group compared with model mice. These findings suggest that the protective effect of XXD on AKI partly results from its antifibrosis, anti-inflammatory, and tubular repair effects.

3.8. Validation of the Hub Genes. The results of bioinformatics analysis were further confirmed by using qRT-PCR on the mRNA levels of the nine hub genes in kidneys of AKI mice which were XXD-treated. Then, to a certain extent, 


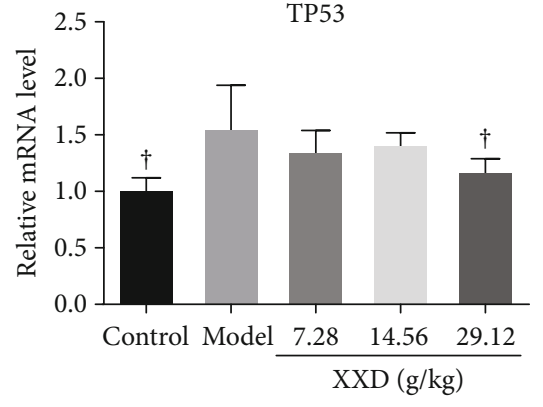

(a)

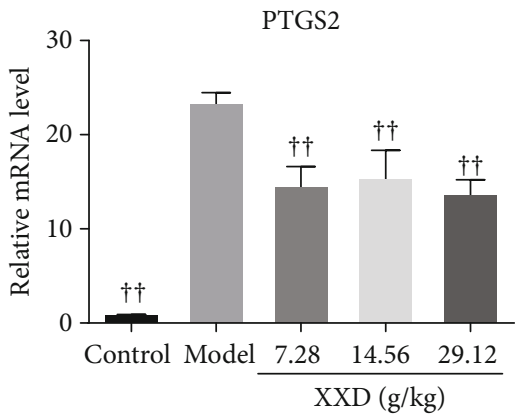

(c)

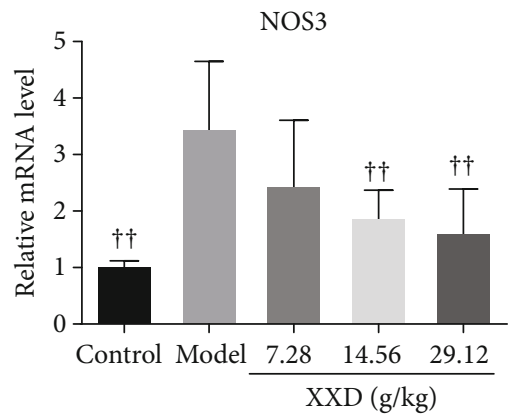

(e)

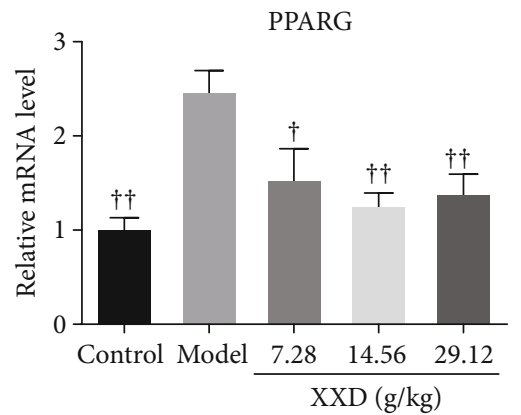

(g)

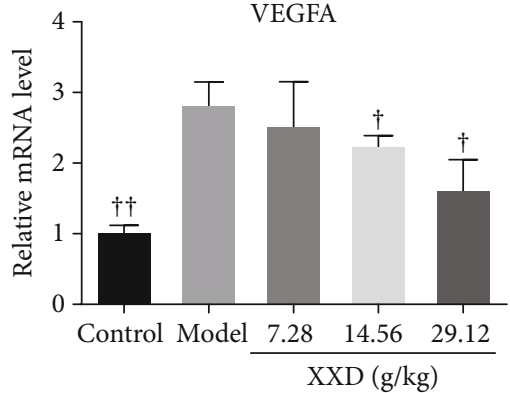

(b)

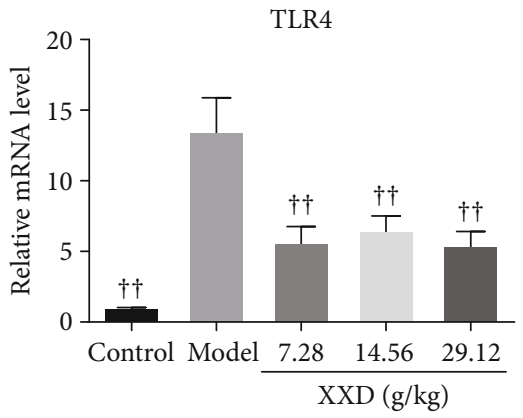

(d)

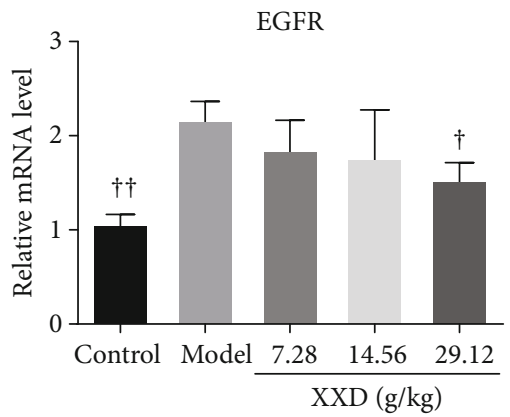

(f)

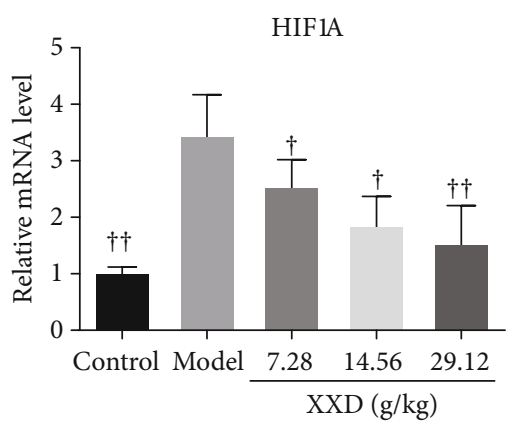

(h)

Figure 9: Continued. 


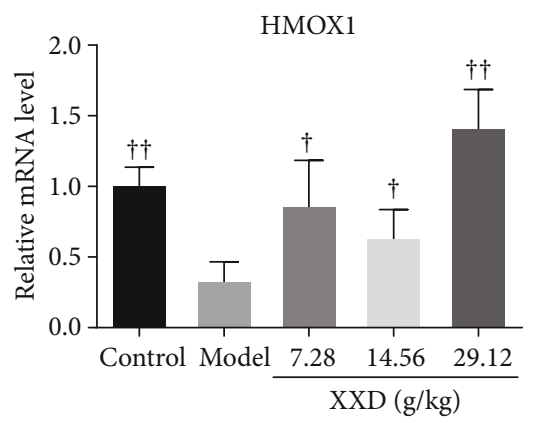

(i)

FIGURE 9: Effect of XXD on the expression levels of hub genes of AKI mice. Quantitative analysis of the expression of (a) TP53, (b) VEGFA, (c) PTGS2, (d) TLR4, (e) NOS3, (f) EGFR, (g) PPARG, (h) HIF1A, and (i) HMOX1. All experiments were performed in triplicate, and results were presented as means $\pm \mathrm{SD}(n=10) .{ }^{\mathbf{\Delta}} P<0.05,{ }^{\mathbf{\Delta}} P<0.01$ vs. the model group.

the qRT-PCR assay showed that the relative expression levels of TP53, VEGFA, PTGS2, TLR4, NOS3, EGFR, PPARG, and HIF1A were significantly decreased in renal tissues of XXD-treated groups (Figures 9(a)-9(h)). Meanwhile, the HMOX1 levels in I/R mouse models treated with XXD were dramatically elevated by XXD (Figure 9(i)).

\subsection{XXD Suppressed TGF- $\beta 1 /$ Smad3 and HIF1 Signaling} Pathways in the Kidneys of I/R AKI Mice. To identify the antifibrosis and antioxidation mechanism of XXD in the kidneys of I/R-induced AKI mice, the TGF- $\beta 1 / \mathrm{Smad} 3$ and HIF1 signaling pathways were further investigated by western blotting analysis. XXD treatment significantly increased MMP-9 expression in mouse kidneys (Figures 10(a) and $10(\mathrm{~b}))$. Also, the protein expression levels of $\alpha$-SMA, ICAM-1, TGF- $\beta 1$, Smad3, p-Smad3, and HIF1A in kidney tissues of AKI mice were decreased after XXD administration (Figures 10(a)-10(d)). Nevertheless, western blotting indicated that high-dose XXD significantly promoted the Smad7 levels in AKI mice (Figures 10(a) and 10(b)).

\section{Discussion}

AKI, a global public health crisis, is associated with significant morbidity, and severe or repeated AKI can transition to CKD or even end-stage renal disease (ESRD), particularly in hospitalized elderly patients $[13,31,32]$. Furthermore, it is well known that ischemia-reperfusion (I/R) injury is a major risk factor for serious clinical problems and is commonly used to investigate the pathogenesis of AKI [33, 34]. Despite significant advances having been made in exploring novel therapeutic strategies, there still remained a lack of effective therapies. Therefore, it is critical to understand more about the underlying mechanism and explore a new approach to treat the disease.

TCM has been widely used in the treatment of varied chronic diseases with multiple targets and multiple pathways. Previous studies have demonstrated the biological properties of TCM in treating AKI, including antioxidant [17], anti-inflammatory [17, 35], and antiapoptosis [36]. In the current study, we successfully identified the absorbable chemical components of Xiaoyu Xiezhuo Drink (XXD) using
UHPLC-Q-TOF-MS analysis. Additionally, the network pharmacology and bioinformatics methods were used to predict component targets and potential biological events in an integral view and promote efficient drug discovery. Based on the compound-target network for XXD, these flavonoids primarily included luteolin, quercetin, isomucronulatol, 3-hydroxy-9,10dimethoxypterocarpan, scutellarein, diosmetin, and naringenin. Luteolin has been reported to ameliorate renal injury and dysfunction in wild-type C57/Bl6 mice with ischemic AKI [37]. Quercetin, a natural flavonoid, widely exists in vegetables, fruits, and Chinese herb medicine. Wang et al. considered that quercetin can protect AKI by inhibiting ferroptosis [38]. Also, Gu et al. found that quercetin may be a promising therapeutic avenue for SARS-CoV-2 injury-induced AKI [39]. Scutellarein is a flavonoid monomer that has been demonstrated to exert various pharmacological effects including anticancer [40], anti-inflammatory [41], and antifibrosis activities [42]. Furthermore, a recent study found that diosmetin also has renoprotective effects in the sepsis-induced AKI model by activating the TUG1/Nrf2/HO-1 pathway [43]. However, as far as we know, people still know little about the effect of isomucronulatol, 3-hydroxy-9,10-dimethoxypterocarpan, and naringenin on I/R-induced AKI. Taken together, these results tell us that flavonoids play an important role in the renoprotective effects of XXD.

In this study, thirty-six crossover genes were determined for drugs and diseases using mapping analysis, which might be targeted for XXD to play an anti-AKI role. Subsequently, GO analysis results showed that the target genes associated with $\mathrm{BP}$ were in response to oxidative stress and the reactive oxygen species metabolic process, etc. Meanwhile, KEGG analysis results showed that the target genes were involved in numerous pathways, and the HIF1 signaling pathway was ranked first. We speculated that these $\mathrm{BP}$ and pathways might be the core physiological events in the treatment of AKI with XXD in elderly men. According to the topological analysis of the PPI network, we further identified 12 hub genes from the 36 crossover genes for the subsequent study including ALB, IL-6, TNF, TP53, VEGFA, PTGS2 (COX-2), TLR4, NOS3 (eNOS), EGFR, PPARG (PPAR- $\gamma$ ), HIF1A, and HMOX1 (HO-1). Among them, TP53 is found to be mutated in approximately half of all tumors and accompanied by increased 


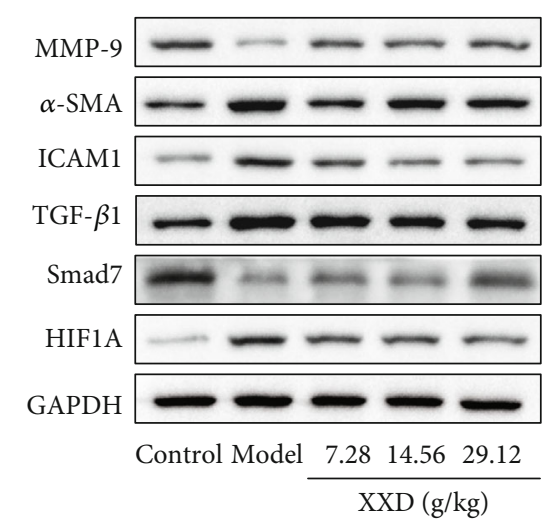

(a)
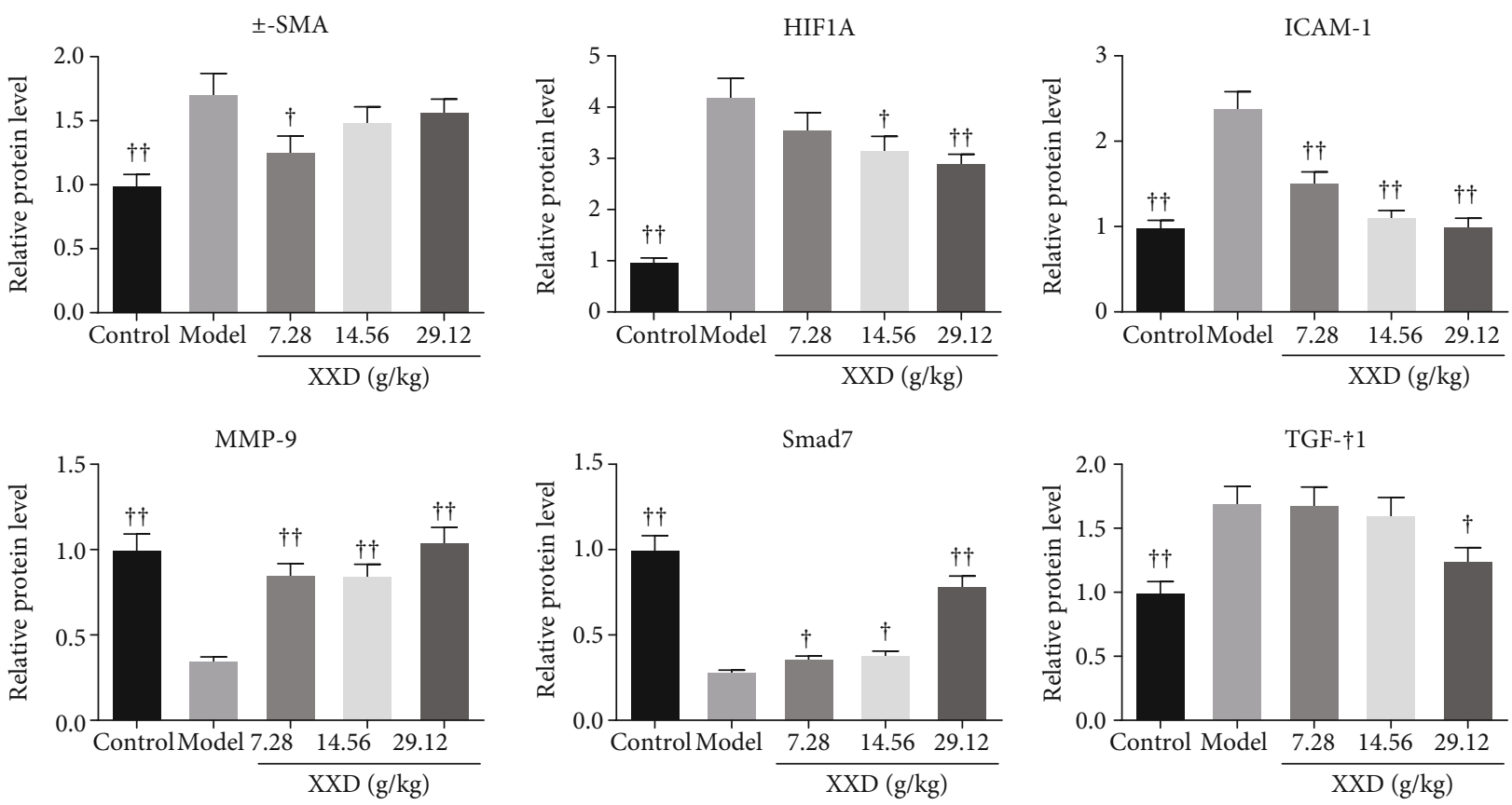

(b)
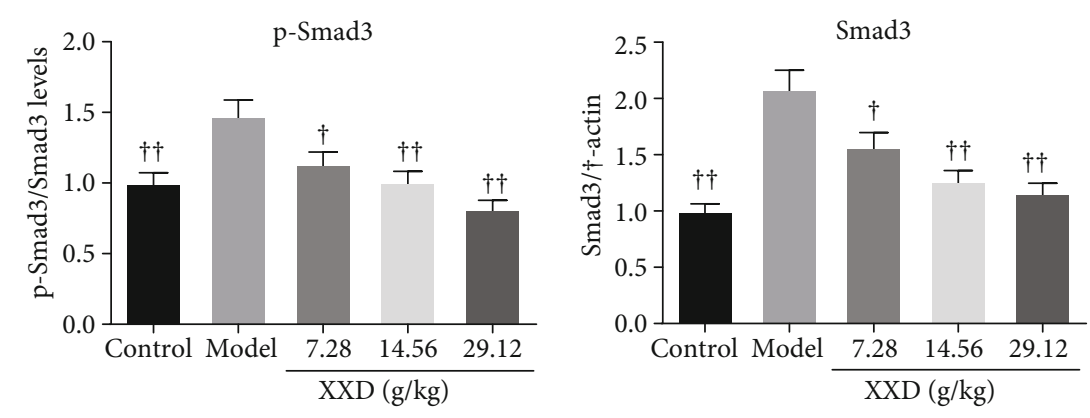

(d)

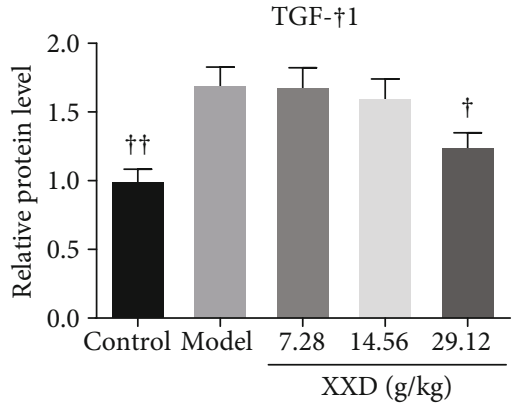

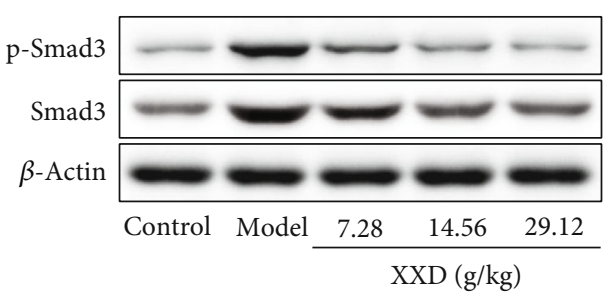

(c)

FIGURE 10: Effect of XXD on the TGF- $\beta 1 / \mathrm{Smad} 3$ and HIF1 signaling pathways of kidney tissues in AKI mice. (a) Representative western blots for MMP-9, $\alpha$-SMA, ICAM-1, TGF- $\beta 1$, Smad7, HIF1A, and GAPDH. Quantitative analysis of (b) MMP-9, $\alpha$-SMA, ICAM-1, TGF$\beta 1$, Smad7, and HIF1A and normalized with GAPDH. (c) Representative protein bands of p-Smad3, Smad3, and $\beta$-actin in XXD-treated AKI mice. (d) Quantitative analysis of $\mathrm{p}$-Smad3 and Smad3 and normalized with $\beta$-actin. All data are represented as the mean \pm SD $(n=3) .{ }^{\boldsymbol{\Delta}} P<0.05,{ }^{\boldsymbol{\Delta}} \mathrm{P}<0.01$ vs. the model group.

expression of TP53 in kidneys of AKI mice [44, 45]. VEGF is widely expressed in the mesenchymal tissue, which promoted the development of vascellum, and possesses proangiogenic effects in renal I/R injury [46]. Furthermore, $\mathrm{Xu}$ et al. found that the expression of VEGFA was significantly increased in AKI patients [2]. COX-2 is positively related to the severity of the inflammatory response and then aggravates kidney tubular epithelial cell injury [47]. TLR4 is one of the members of the 
Toll-like receptor (TLR) family, which can induce inflammatory cytokine expression, and then lead to inflammation reaction with consequent renal injury [48]. Endothelial nitric oxide synthase (eNOS) belongs to the isozymes of nitric oxide (NO) synthase and is distributed in vascular endothelial cells in the kidney. Also, Ohkita et al. demonstrated that the ameliorating effects of the grape extract existed in wild-type mice, but not in eNOS knockout mice [49]. For the EGFR, the role of EGFR in AKI may be controversial. A recent study has shown that EGFR plays a detrimental role in I/R-induced AKI [50]. Nonetheless, other studies suggest that EGFR is responsible for the renoprotective role on the renal fibrosis of $\mathrm{I} / \mathrm{R}$ injury $[51,52]$. Recently, Singh et al. reported that I/R injury caused a significant decrease in the levels of PPAR- $\gamma$ and eNOS in rat kidneys [53]. It suggests that PPAR- $\gamma$ activation may be associated with inhibition of oxidative stress and subsequent improvement of AKI. HIF1A, an oxygen-sensitive subunit, is stable under hypoxic environments. To the best of our knowledge, there are no studies that have evaluated the effect of TCM on HIF1A in I/Rinduced AKI aged mouse models. HMOX1 is a cytoprotective enzyme characterized by its antiapoptotic and antiinflammatory properties to protect against AKI [54]. As discussed above, the roles of hub genes on the processes of AKI must be further examined.

As widely accepted, I/R injury-induced AKI is characterized by inflammation, oxidative stress, and a sharp rise of serum creatinine and BUN levels. In the current study, the nephroprotective effects of XXD were investigated using an I/R injuryinduced AKI aged mouse model. Interestingly, we observed that XXD significantly decreased the serum Cre, BUN, urinary ALB, and inflammatory factor levels and enhanced the antioxidant activities of the kidney in AKI aged mice. Moreover, XXD effectively improved the histological changes, collagen deposition, and cell senescence in renal tissues of AKI mice. Our study also demonstrated XXD treatment ameliorating kidney fibrosis and promoting cellular proliferation, as accompanied by attenuating the protein expression of $\alpha$-SMA, collagen-1, F4/80, and ICAM-1, as well as dramatically increasing the p-H3, Ki67, and MMP-9 levels. Additionally, the qPCR analysis demonstrated that the TP53, VEGFA, PTGS2, TLR4, NOS3, EGFR, PPARG, and HIF1A were downregulated, while HMOX1 was found to be upregulated in XXD-treated AKI aged mice. TGF- $\beta 1$ has been reported to promote fibrosis in the kidney with I/R injury [13]. Furthermore, a recent study demonstrated that the knockout of Smad3 attenuated I/R-induced AKI in diabetic kidney by interacting with p53 and NOX4 [55]. Additionally, many studies have shown that overexpression of Smad7 caused the inhibition of renal inflammation by inhibiting NF$\kappa \mathrm{B}$ activation $[56,57]$. Smad7, a negative regulator of TGF- $\beta$ signaling, is able to compete with Smad3, in order to inhibit the phosphorylation of receptor-activated Smads [58]. In this regard, it is worth noting the effects of XXD on the TGF$\beta /$ Smads in AKI aged mice. As we have shown, we also found that there was downregulation of TGF- $\beta 1, \operatorname{Smad} 3, \operatorname{Smad} 3$ phosphorylation, and elevated Smad7 protein levels after XXD treatment in AKI aged mice. Taken together, these investigations suggested that XXD exerted its renoprotective effects via ameliorating inflammation and oxidative stress by inhibiting the TGF- $\beta 1 /$ Smad3 and HIF1 signaling pathways.

\section{Conclusion}

In summary, the pharmacological mechanism of XXD to ameliorate ischemic AKI was explored with the combination of network pharmacology prediction and experimental validation. By constructing component-target and PPI networks, the flavonoids and several hub genes such as luteolin, quercetin, scutellarein, diosmetin, TP53, VEGFA, PTGS2, TLR4, NOS3, EGFR, PPARG, HIF1A, and HMOX1 might be promising for the treatment of AKI. Furthermore, We discovered that the effects of XXD may be associated with its anti-inflammatory and antioxidant activities in I/R injury-induced AKI aged mice via the regulation of TGF- $\beta 1 / \mathrm{Smad} 3$ and HIF1 signaling pathways, which provide new insights for strengthening the understanding of the therapeutic effects of XXD on AKI. However, to confirm the effects of XXD, further experimental experiments are needed to validate these results in various AKI models and large-scale clinical trials.

\begin{tabular}{|c|c|}
\hline AKI: & Acute kidney injury \\
\hline XXD: & Xiaoyu Xiezhuo Drink \\
\hline UHPLC-Q-TOF-MS: & $\begin{array}{l}\text { Ultra-high-performance liquid chro- } \\
\text { matography coupled with hybrid tri- } \\
\text { ple quadrupole time-of-flight mass } \\
\text { spectrometry }\end{array}$ \\
\hline PPI: & Protein-protein interaction \\
\hline I/R: & Ischemia-reperfusion \\
\hline ARF: & Acute renal failure \\
\hline GFR: & Glomerular filtration rate \\
\hline CKD: & Chronic kidney disease \\
\hline IL-6: & Interleukin 6 \\
\hline TNF- $\alpha:$ & Tumor necrosis factor $\alpha$ \\
\hline ICAM-1: & Intercellular adhesion molecule 1 \\
\hline TGF- $\beta$ : & Transforming growth factor $\beta$ \\
\hline MMP-7/9: & Metalloproteinase $7 / 9$ \\
\hline TCM: & Traditional Chinese medicine \\
\hline$\alpha$-SMA: & $\alpha$-Smooth muscle actin \\
\hline UUO: & Unilateral ureteral occlusion. \\
\hline
\end{tabular}

\section{Data Availability}

The data used to support the findings of this study are available from the corresponding author upon request.

\section{Conflicts of Interest}

The authors declare that they have no conflicts of interest.

\section{Authors' Contributions}

Qingqing Ye, Cong Xia, Shouci Hu, and Hongbo Chen conducted all experiments; Qingqing Ye collected the data; Lanjun Fu analyzed the results; and Qingqing Ye, Hongzhen $\mathrm{Ma}$, and Xiaojun Xiang wrote the manuscript; all authors reviewed and approved the manuscript. 


\section{Acknowledgments}

This work was funded by the Program of Zhejiang Provincial TCM Sci-Tech Plan (No. 2019ZQ021, No. 2020ZB075, and No. 2021ZB110).

\section{Supplementary Materials}

Supplementary 1. Table S1: high-resolution mass spectrometry data and elemental composition of XXD (positive ion mode).

Supplementary 2. Table S2: high-resolution mass spectrometry data and elemental composition of XXD (negative ion mode).

\section{References}

[1] X. Liu, R. Zhang, L. Huang et al., "Excessive oxidative stress contributes to increased acute ER stress kidney injury in aged mice," Oxidative Medicine and Cellular Longevity, vol. 2019, Article ID 2746521, 15 pages, 2019.

[2] Y. Xu, W. Jiang, L. Zhong et al., "miR-195-5p alleviates acute kidney injury through repression of inflammation and oxidative stress by targeting vascular endothelial growth factor A," Aging (Albany NY), vol. 12, no. 11, article 103160, pp. 10235-10245, 2020.

[3] A. Goyal, P. Daneshpajouhnejad, M. F. Hashmi, and K. Bashir, Acute kidney injury, StatPearls Publishing, Treasure Island (FL), 2021.

[4] Y. Wang, J. Cai, C. Tang, and Z. Dong, "Mitophagy in acute kidney injury and kidney repair," Cell, vol. 9, no. 2, p. 338, 2020.

[5] A. Zuk and J. V. Bonventre, "Acute kidney injury," Annual Review of Medicine, vol. 67, no. 1, pp. 293-307, 2016.

[6] J. Barasch, R. Zager, and J. V. Bonventre, "Acute kidney injury: a problem of definition," Lancet, vol. 389, no. 10071, pp. 779781, 2017.

[7] L. Yang, G. Xing, L. Wang et al., "Acute kidney injury in China: a cross-sectional survey," Lancet, vol. 386, no. 10002, pp. 1465-1471, 2015.

[8] J. Cui, X. Bai, and X. Chen, "Autophagy and acute kidney injury," Advances in Experimental Medicine and Biology, vol. 1207, pp. 469-480, 2020.

[9] J. T. Kurzhagen, S. Dellepiane, V. Cantaluppi, and H. Rabb, "AKI: an increasingly recognized risk factor for CKD development and progression," Journal of Nephrology, vol. 33, no. 6, pp. 1171-1187, 2020.

[10] H. E. Wheeler and S. K. Kim, "Genetics and genomics of human ageing," Philosophical Transactions of the Royal Society of London. Series B, Biological Sciences, vol. 366, no. 1561, pp. 43-50, 2011.

[11] P. He, D. Li, and B. Zhang, "Losartan attenuates renal interstitial fibrosis and tubular cell apoptosis in a rat model of obstructive nephropathy," Molecular Medicine Reports, vol. 10, no. 2, pp. 638-644, 2014.

[12] J. V. Bonventre and L. Yang, "Cellular pathophysiology of ischemic acute kidney injury," Journal of Clinical Investigation, vol. 121, no. 11, pp. 4210-4221, 2011.

[13] N. Arfian, D. A. P. Wahyudi, I. B. Zulfatina et al., "Chlorogenic acid attenuates kidney ischemic/reperfusion injury via reducing inflammation, tubular injury, and myofibroblast formation," BioMed Research International, vol. 2019, Article ID 5423703, 10 pages, 2019.
[14] J. M. van Deursen, "The role of senescent cells in ageing," Nature, vol. 509, no. 7501, pp. 439-446, 2014.

[15] L. Andrade, C. E. Rodrigues, S. A. Gomes, and I. L. Noronha, "Acute kidney injury as a condition of renal senescence," Cell Transplantation, vol. 27, no. 5, pp. 739-753, 2018.

[16] H. D. Li, X. M. Meng, C. Huang, L. Zhang, X. W. Lv, and J. Li, "Application of herbal traditional Chinese medicine in the treatment of acute kidney injury," Frontiers in Pharmacology, vol. 10, p. 376, 2019.

[17] M. S. Islam, L. Miao, H. Yu, Z. Han, and H. Sun, "Ethanol extract of Illicium henryi attenuates LPS-induced acute kidney injury in mice via regulating inflammation and oxidative stress," Nutrients, vol. 11, no. 6, p. 1412, 2019.

[18] K. LU, Z. XIE, and M. A. Hongzhen, "Effects of Xiaoyu Xiezhuo Yin on the expression of TGF- $\beta 1$ of renal tissue in rat with UUO," Chinese Journal of Integrated Traditional and Western Nephrology, vol. 12, no. 8, pp. 665-668, 2011.

[19] K.-d. LU, B.-b. ZHANG, Z.-j. XIE, M. A. Hong-zhen, H. E. Ling-zhi, and L. I. Xue-ming, "The protective effect of Xiaoyu Xiezhuo Yin on kidney of renal interstitial fibrosis rats," Chinese Archives of Traditional Chinese Medicine, vol. 7, no. 29, pp. 1537-1540, 2011.

[20] A. L. Hopkins, "Network pharmacology," Nature Biotechnology, vol. 25, no. 10, pp. 1110-1111, 2007.

[21] Z. Zhou, B. Chen, S. Chen et al., "Applications of network pharmacology in traditional Chinese medicine research," Evidence-based Complementary and Alternative Medicine, vol. 2020, Article ID 1646905, 7 pages, 2020.

[22] S. Kim, J. Chen, T. Cheng et al., "PubChem in 2021: new data content and improved web interfaces," Nucleic Acids Research, vol. 49, no. D1, pp. D1388-D1395, 2021.

[23] A. Daina, O. Michielin, and V. Zoete, "SwissTargetPrediction: updated data and new features for efficient prediction of protein targets of small molecules," Nucleic acids research, vol. 47, no. W1, pp. W357-W364, 2019.

[24] D. Szklarczyk, A. Santos, C. von Mering, L. J. Jensen, P. Bork, and M. Kuhn, "STITCH 5: augmenting protein-chemical interaction networks with tissue and affinity data," Nucleic Acids Research, vol. 44, no. D1, pp. D380-D384, 2016.

[25] P. Shannon, A. Markiel, O. Ozier et al., "Cytoscape: a software environment for integrated models of biomolecular interaction networks," Genome Research, vol. 13, no. 11, pp. 24982504, 2003.

[26] J. H. Wang, L. F. Zhao, H. F. Wang et al., "GenCLiP 3: mining human genes' functions and regulatory networks from PubMed based on co-occurrences and natural language processing," Bioinformatics, vol. 36, no. 6, pp. 1973-1975, 2020.

[27] G. Stelzer, N. Rosen, I. Plaschkes et al., "The GeneCards suite: from gene data mining to disease genome sequence analyses," Current Protocols in Bioinformatics, vol. 54, no. 1, pp. 1.30.11.30.33, 2016.

[28] J. Piñero, J. M. Ramírez-Anguita, J. Saüch-Pitarch et al., "The DisGeNET knowledge platform for disease genomics: 2019 update," Nucleic Acids Research, vol. 48, no. D1, pp. D845D855, 2020.

[29] Y. Zhou, B. Zhou, L. Pache et al., "Metascape provides a biologist-oriented resource for the analysis of systems-level datasets," Nature Communications, vol. 10, no. 1, p. 1523, 2019.

[30] D. Szklarczyk, A. L. Gable, D. Lyon et al., "STRING v11: protein-protein association networks with increased coverage, 
supporting functional discovery in genome-wide experimental datasets," Nucleic Acids Research, vol. 47, no. D1, pp. D607D613, 2019.

[31] M. Formica, P. Politano, F. Marazzi et al., "Acute kidney injury and chronic kidney disease in the elderly and polypharmacy," Blood Purification, vol. 46, no. 4, pp. 332-336, 2018.

[32] L. Gao, X. Zhong, J. Jin, J. Li, and X. M. Meng, "Potential targeted therapy and diagnosis based on novel insight into growth factors, receptors, and downstream effectors in acute kidney injury and acute kidney injury-chronic kidney disease progression," Signal Transduction and Targeted Therapy, vol. 5, no. 1, p. 9, 2020.

[33] J. Tang and S. Zhuang, "Histone acetylation and DNA methylation in ischemia/reperfusion injury," Clinical Science (London, England), vol. 133, no. 4, pp. 597-609, 2019.

[34] A. J. Polichnowski, K. A. Griffin, H. Licea-Vargas et al., "Pathophysiology of unilateral ischemia-reperfusion injury: importance of renal counterbalance and implications for the AKICKD transition," American Journal of Physiology. Renal Physiology, vol. 318, no. 5, pp. F1086-F1099, 2020.

[35] B. Zhang, M. Zeng, M. Li et al., "Protopine protects mice against LPS-induced acute kidney injury by inhibiting apoptosis and inflammation via the TLR4 signaling pathway," Molecules, vol. 25, no. 1, p. 15, 2020.

[36] D. Xu, M. Chen, X. Ren, X. Ren, and Y. Wu, "Leonurine ameliorates LPS-induced acute kidney injury via suppressing ROSmediated NF- $\kappa$ B signaling pathway," Fitoterapia, vol. 97, pp. 148-155, 2014.

[37] A. Andres-Hernando, N. Li, C. Cicerchi et al., "Protective role of fructokinase blockade in the pathogenesis of acute kidney injury in mice," Nature Communications, vol. 8, no. 1, article 14181, 2017.

[38] Y. Wang, F. Quan, Q. Cao et al., "Quercetin alleviates acute kidney injury by inhibiting ferroptosis," Journal of Advanced Research, vol. 28, pp. 231-243, 2021.

[39] Y. Y. Gu, M. Zhang, H. Cen et al., "Quercetin as a potential treatment for COVID-19-induced acute kidney injury: based on network pharmacology and molecular docking study," PLoS One, vol. 16, no. 1, article e0245209, 2021.

[40] Y. Li, J. Wang, S. Zhong, J. Li, and W. du, "Scutellarein inhibits the development of colon cancer via CDC4-mediated RAGE ubiquitination," International Journal of Molecular Medicine, vol. 45, no. 4, pp. 1059-1072, 2020.

[41] N. Y. Sung, M. Y. Kim, and J. Y. Cho, "Scutellarein reduces inflammatory responses by inhibiting Src kinase activity," The Korean journal of physiology \& pharmacology, vol. 19, no. 5, pp. 441-449, 2015.

[42] K. Miao, T. Pan, Y. Mou et al., "Scutellarein inhibits BLMmediated pulmonary fibrosis by affecting fibroblast differentiation, proliferation, and apoptosis," Therapeutic Advances in Chronic Disease, vol. 11, 2020.

[43] W. Wang, S. Zhang, F. Yang, J. Xie, J. Chen, and Z. Li, “Diosmetin alleviates acute kidney injury by promoting the TUG1/Nrf2/HO-1 pathway in sepsis rats," International Immunopharmacology, vol. 88, article 106965, 2020.

[44] D. Zhou, Y. Li, L. Lin, L. Zhou, P. Igarashi, and Y. Liu, "Tubule-specific ablation of endogenous $\beta$-catenin aggravates acute kidney injury in mice," Kidney International, vol. 82, no. 5, pp. 537-547, 2012.

[45] R. P. Kaur, K. Vasudeva, R. Kumar, and A. Munshi, "Role of p53 gene in breast cancer: focus on mutation spectrum and therapeutic strategies," Current Pharmaceutical Design, vol. 24, no. 30, pp. 3566-3575, 2018.

[46] L. Yuan, M. J. Wu, H. Y. Sun et al., "VEGF-modified human embryonic mesenchymal stem cell implantation enhances protection against cisplatin-induced acute kidney injury," American Journal of Physiology. Renal Physiology, vol. 300, no. 1, pp. F207-F218, 2011.

[47] H. Fan, J. W. le, M. Sun, and J. H. Zhu, "Pretreatment with Snitrosoglutathione attenuates septic acute kidney injury in rats by inhibiting inflammation, oxidation, and apoptosis," BioMed Research International, vol. 2021, Article ID 6678165, 8 pages, 2021.

[48] J. S. Deng, W. P. Jiang, C. C. Chen et al., "Cordyceps cicadae mycelia ameliorate cisplatin-induced acute kidney injury by suppressing the TLR4/NF- $\kappa \mathrm{B} / \mathrm{MAPK}$ and activating the HO1/Nrf2 and Sirt-1/AMPK pathways in mice," Oxidative Medicine and Cellular Longevity, vol. 2020, Article ID 7912763, 17 pages, 2020.

[49] M. Ohkita, H. Hayashi, K. Ito et al., "Preventive effects of grape extract on ischemia/reperfusion-induced acute kidney injury in mice," Biological \& Pharmaceutical Bulletin, vol. 42, no. 11, pp. 1883-1890, 2019.

[50] L. Xu, X. Li, F. Zhang, L. Wu, Z. Dong, and D. Zhang, "EGFR drives the progression of AKI to CKD through HIPK2 overexpression," Theranostics, vol. 9, no. 9, pp. 2712-2726, 2019.

[51] X. Xu, J. Wang, R. Yang, Z. Dong, and D. Zhang, "Genetic or pharmacologic inhibition of EGFR ameliorates sepsisinduced AKI," Oncotarget, vol. 8, no. 53, article 21244, pp. 91577-91592, 2017.

[52] S. He, N. Liu, G. Bayliss, and S. Zhuang, "EGFR activity is required for renal tubular cell dedifferentiation and proliferation in a murine model of folic acid-induced acute kidney injury," American Journal of Physiology. Renal Physiology, vol. 304, no. 4, pp. F356-F366, 2013.

[53] A. P. Singh, N. Singh, D. Pathak, and P. M. S. Bedi, "Estradiol attenuates ischemia reperfusion-induced acute kidney injury through PPAR- $\gamma$ stimulated eNOS activation in rats," Molecular and Cellular Biochemistry, vol. 453, no. 1-2, pp. 1-9, 2019.

[54] O. Adedoyin, R. Boddu, A. Traylor et al., "Heme oxygenase-1 mitigates ferroptosis in renal proximal tubule cells," American Journal of Physiology. Renal Physiology, vol. 314, no. 5, pp. F702-F714, 2018.

[55] J. N. Wang, Q. Yang, C. Yang et al., "Smad3 promotes AKI sensitivity in diabetic mice via interaction with p53 and induction of NOX4-dependent ROS production," Redox Biology, vol. 32, article 101479, 2020.

[56] C. Yang, Y. Guo, T. S. Huang et al., "Asiatic acid protects against cisplatin-induced acute kidney injury via antiapoptosis and anti-inflammation," Biomedicine \& Pharmacotherapy, vol. 107, pp. 1354-1362, 2018.

[57] Z. Li, Z. Hong, Z. Peng, Y. Zhao, and R. Shao, “Acetylshikonin from Zicao ameliorates renal dysfunction and fibrosis in diabetic mice by inhibiting TGF- $\beta 1 /$ Smad pathway," Human Cell, vol. 31, no. 3, pp. 199-209, 2018.

[58] J. Ji and L. He, "Effect of Kangxianling decoction on expression of TGF- $\beta 1 /$ Smads and extracellular matrix deposition," Evidence-based Complementary and Alternative Medicine, vol. 2019, Article ID 5813549, 9 pages, 2019. 